\title{
Quantum-state preparation and macroscopic entanglement in gravitational-wave detectors
}

\author{
Helge Müller-Ebhardt, ${ }^{1}$ Henning Rehbein, ${ }^{1}$ Chao Li, ${ }^{2}$ Yasushi Mino, ${ }^{2}$ Kentaro Somiya, ${ }^{3}$ Roman Schnabel, ${ }^{1}$ \\ Karsten Danzmann, ${ }^{1}$ and Yanbei Chen ${ }^{2,3}$ \\ ${ }^{1}$ Max-Planck-Institut für Gravitationsphysik (Albert-Einstein-Institut), Institut für Gravitationsphysik, Leibniz Universität Hannover, \\ Callinstr. 38, 30167 Hannover, Germany \\ ${ }^{2}$ California Institute of Technology, M/C 130-33, Pasadena, California 91125, USA \\ ${ }^{3}$ Max-Planck-Institut für Gravitationsphysik (Albert-Einstein-Institut), Am Mühlenberg 1, 14476 Potsdam, Germany
}

(Received 28 February 2009; published 2 October 2009)

\begin{abstract}
Long-baseline laser-interferometer gravitational-wave $(\mathrm{GW})$ detectors are operating at a factor of $\sim 10$ (in amplitude) above the standard quantum limit (SQL) within a broad frequency band (in the sense that $\Delta f \sim f$ ). Such a low-noise budget has already allowed the creation of a controlled $2.7 \mathrm{~kg}$ macroscopic oscillator with an effective eigenfrequency of $150 \mathrm{~Hz}$ and an occupation number of $\sim 200$. This result, along with the prospect for further improvements, heralds the possibility of experimentally probing macroscopic quantum mechanics (MQM) - quantum mechanical behavior of objects in the realm of everyday experience-using GW detectors. In this paper, we provide the mathematical foundation for the first step of a MQM experiment: the preparation of a macroscopic test mass into a nearly minimum-Heisenberg-limited Gaussian quantum state, which is possible if the interferometer's classical noise beats the SQL in a broad frequency band. Our formalism, based on Wiener filtering, allows a straightforward conversion from the noise budget of a laser interferometer, in terms of noise spectra, into the strategy for quantum-state preparation and the quality of the prepared state. Using this formalism, we consider how Gaussian entanglement can be built among two macroscopic test masses and the performance of the planned Advanced LIGO interferometers in quantum-state preparation.
\end{abstract}

DOI: $10.1103 /$ PhysRevA.80.043802

PACS number(s): 42.50.Dv, 42.50.Xa, 42.50.Lc, 03.65.Ta

\section{INTRODUCTION}

There is an international array of first-generation longbaseline laser interferometric gravitational-wave (GW) detectors (LIGO [1], VIRGO [2], GEO [3], and TAMA [4]) spanned all over the world. These detectors are now reaching their design sensitivities. They all consist of Michelson interferometers and have been built to measure GW-driven relative length changes (within a detection band from $10 \mathrm{~Hz}$ to $10 \mathrm{kHz}$ ) between the mirror-endowed test masses which are hung as pendulums with an eigenfrequency far below the detection band. Resonant cavities are used to enhance the sensitivity by increasing the circulating optical power and the signal storage time. In Michelson interferometers usually the total differential mode of motion between the arm-cavity mirrors, in the following always represented by the position operator

$$
\hat{x}=\left(\hat{x}_{\mathrm{TTM}_{\mathrm{E}}}-\hat{x}_{\mathrm{ETM}_{\mathrm{E}}}\right)-\left(\hat{x}_{\mathrm{ITM}_{\mathrm{N}}}-\hat{x}_{\mathrm{ETM}_{\mathrm{N}}}\right)
$$

(for the nomenclature see Fig. 1), is measured via a homodyne detection of the modulation fields (also called sideband fields) leaking out at the dark port of the interferometer. Current GW interferometers are already quantum limited at high frequencies by the shot noise. Next generation interferometers, such as the planned Advanced LIGO detector [5] (cf. Fig. 1), are expected to be quantum noise limited at nearly all frequencies in the detection band. Advanced LIGO will therefore operate at its free-mass standard quantum limit (SQL) [6-8] at which the back-action noise is comparable to the shot noise level. Generally, the SQL is the minimum noise spectrum achievable by a linear quantum measurement with uncorrelated sensing and back-action noise. The position-referred spectral density of the SQL at the sideband frequency $\Omega$ is given by

$$
S_{\mathrm{SQL}}(\Omega) \equiv \frac{2 \hbar}{m \Omega^{2}} .
$$

Here $m$ is the reduced mass of the measured object which in the case of (Advanced) LIGO is $1 / 4$ of the individual armcavity mirror mass.

Improvement of sensitivities to GWs beyond Advanced LIGO will require to surpass the SQL significantly in a broad frequency band. Various conceptual strategies exist for build-

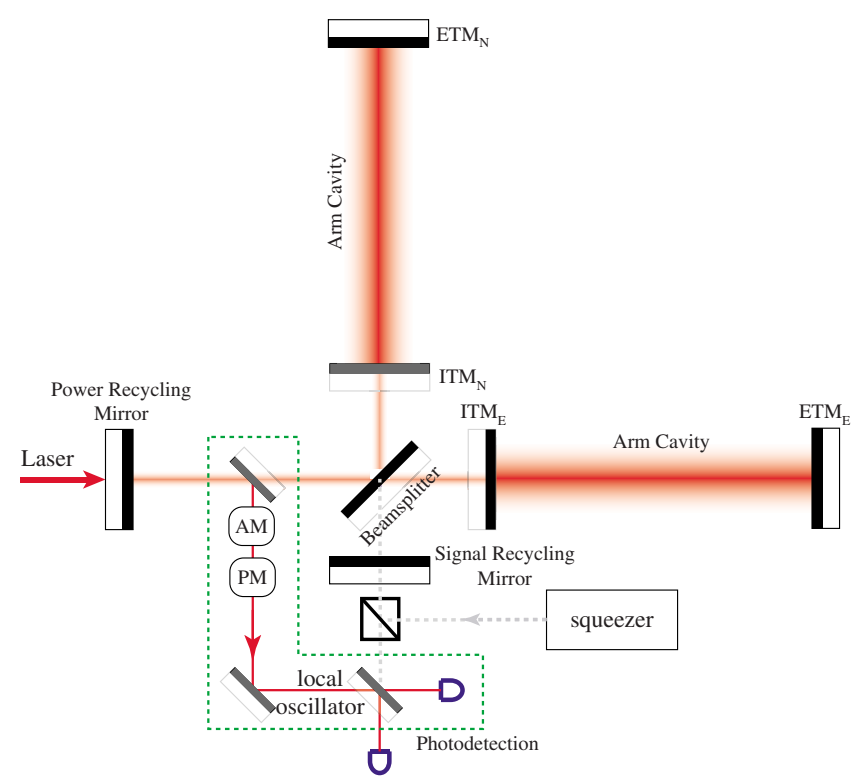

FIG. 1. (Color online) Schematic plot of the planned Advanced LIGO [5] interferometer: a power- and a signal-recycled Michelson interferometer with cavities in the arms and a homodyne detection scheme at the dark port. 
ing interferometers with broadband sub-SQL quantum noise (see, e.g., Refs. [9-24]), while there is also much effort devoted to lowering classical noise below the SQL. For example, (i) the CLIO interferometer is currently being cooled down to a few tens of kelvin and has a theoretical thermal noise budget below the SQL [25], (ii) nonspherical mirrors are being developed that support non-Gaussian modes which average better over mirror surfaces and are thus less susceptible to thermal noise [26-28], and (iii) coating structures are also being optimized for lowering the coating thermal noise [29,30].

This paper, however, is devoted not to the improvement of the detector's sensitivity to GWs but to the study of quantum mechanical behavior of its test masses. Recently, it has been reported by the LIGO Scientific Collaboration in Ref. [31] that a certain (controlled) mode of the mirror's differential motion in the LIGO detector located in Hanford was experimentally cooled down to $1.4 \mu \mathrm{K}$, with an effective occupation number of around 200. Furthermore, there are a number of other experiments considering different smaller-scale mechanical structures (see, e.g., Refs. [32-39]). The goal of all of those cold damping [40] experiments is to reach the oscillator's ground state. Those experiments consider a certain controlled, but nonselective (or unconditional), state of the test mass. But as it is shown in Ref. [41], the controlled unconditional state of a system is always more mixed than the corresponding selective (or conditional) state. Other state preparation experiments consider also the unconditional state of the test mass without using an active feedback control but using only the back action of the measurement (see, e.g., Refs. [42-45]), which is usually called self-cooling [46]. The in such a way prepared states are also always more mixed than their conditional counterparts.

One aim of this paper is to provide a mathematical foundation guiding future experimental efforts of quantum-state preparation-one that is straightforward to apply to experimental situations. Our formulation is different in the context of quantum-state preparation attempts using a continuous position measurement of an oscillator test mass. Our approach relies on a frequency-domain treatment, which is more suitable, because the characteristic spectra in an experiment are usually easily accessible. The oscillator is only specified by its mass, eigenfrequency, and the linear measurement only by the noise spectrum. Quantum mechanically, we consider a problem in which an object is being continuously measured, while it is simultaneously subject to noisy forces. For this problem, the stochastic master equation (SME) (as used in, e.g., Refs. [47-50]) is in fact a readily available tool to simulate quantum-state preparation in quantum mechanical systems: a Riccati equation associated with the SME provides the remaining uncertainty of the object when all measurement data are taken into account. Formally, this approach only treats Markovian systems, while non-Markovian is prevalent in experiments such as in GW detectors: virtually none of the noise sources are white, and we have the added complexity that the cavity mode often couples strongly with the test masses, i.e., it cannot be adiabatically eliminated [50], and must be evolved together with the test masses. If we only consider linear systems (i.e., systems whose Hamiltonian is at most quadratic in its canonical coordinates and momenta [51]) with Gaussian noise and we only care about the test masses' state after the initial transient has died down, the SME and the Riccati equation, which also characterizes the exact way of the transient decays, are not entirely necessary. In Refs. $[47,48]$ it was already realized that there is an analogy between the SME and Kalman filtering [52]. In the steady state, we have found that a Wiener filtering approach, in which the measurement data are filtered with the optimal causal Wiener filter [53] to obtain instantaneous optimal estimates for position and momentum of the test masses [54], suffices and is most straightforwardly connected with experimental calibration of the system. This is the root of our approach which applies to all stable linear Gaussian systems under linear continuous measurement of a proper measurement device.

A direct application of the Wiener filtering approach is to explore how quantum a macroscopic test mass can be prepared in a certain environment, which is the second aim of this paper. We measure the purity of the Gaussian quantum state of an individual test mass (or a single mechanical degree of freedom) through the Heisenberg uncertainty product or the following quantity:

$$
U \equiv \frac{2}{\hbar} \sqrt{V_{x x} V_{p p}-V_{x p}^{2}} \geq 1,
$$

which is unity for a pure state. This quantity is simply connected to the linear entropy and can also be converted into an effective occupation number as shown in Appendix A. Here $V_{x x}, V_{p p}$, and $V_{x p}$ are the second-order moments of position and momentum of the Gaussian state. We will show that a low classical-noise budget which is completely below the SQL for a broad frequency band allows the quantity $U$ to become close to unity. In particular for simple systems with a total classical-noise spectrum $S_{\mathrm{cl}}(\Omega)$ which is dominated by a white sensing noise and a white force, we have obtained the simple relation

$$
U \approx 1+\min _{\Omega}\left\{\frac{S_{\mathrm{cl}}(\Omega)}{S_{\mathrm{SQL}}(\Omega)}\right\} .
$$

While our discussion and our results are at the beginning general, in the course of the paper we will specify to suspended mirrors with very low eigenfrequencies which are optically read out and, moreover, we will specifically treat the differential mode of motion between the $\mathrm{kg}$-scale mirrors of the Advanced LIGO detector in the end. We shall explore how a realistic noise budget for the planned Advanced LIGO detector-as well as an extension of Advanced LIGO with plausible improvement-can best be taken advantage of through an optimized optical configuration that minimizes $U$. Furthermore, when two independent mechanical degrees of freedom are considered, as two suspended mirrors in a Michelson interferometer, the formalism, which we present in this paper, has already been applied to show that the production of quantum entanglement between the macroscopic end mirrors is possible for sub-SQL laser interferometers [54].

Note that an experiment testing macroscopic quantum mechanics (MQM) should be divided into different stages which are separated in time: a preparation stage, where the 
test mass will be continuously observed; an optional freeevolution stage; and a verification stage. One will need to collect statistics from a huge number of identical trials as it is required from quantum mechanics. This present paper is therefore only the first one of a paper series: this one deals with the preparation of macroscopic conditional quantum states; while a second paper [55] will study the verification of such macroscopic quantum states.

This paper is organized as follows: in Sec. II we will briefly review the theoretical basics of Wiener filtering and how it applies to our problem. In Sec. III we will study analytically the conditional variances of a simplified model using only Markovian dynamics. We start with a general treatment in Sec. III A and then specify to an interferometric measurement in Secs. III B and III C, where we will introduce a flexible homodyne detection angle and input squeezing. In Sec. IV we will extend the analysis done in Ref. [54] about macroscopic entanglement. In Sec. V we will study test masses in a cavity with finite bandwidth and we will treat more realistic non-Markovian noise sources. These preliminary studies result in an investigation of quantum-state preparation in Advanced LIGO in Sec. VI. Finally, in Sec. VII we will summarize our main conclusions.

\section{WIENER FILTERING}

For systems under continuous measurement, the conventional approach is to describe the joint system-measurementdata (selective) evolution using a SME [50], which is a set of stochastic differential equations that simulates the joint evolution of the system's conditional density matrix $\hat{\rho}^{\text {cond }}$ and measurement data $y(t)$. As the simplest example, for a harmonic oscillator with position $\hat{x}$ being measured continuously by a Markovian measurement device (one that has uncorrelated measurement noise at different times and constant measurement strength), the SME reads

$$
\begin{gathered}
d \hat{\rho}^{\mathrm{cond}}=-\frac{i}{\hbar}\left[H_{\mathrm{sys}}, \hat{\rho}^{\mathrm{cond}}\right] d t-\frac{\alpha^{2}}{4 \hbar^{2}}\left[\hat{x},\left[\hat{x}, \hat{\rho}^{\mathrm{cond}}\right]\right] d t \\
+\frac{\alpha}{\sqrt{2} \hbar}\left(\left\{\hat{x}, \hat{\rho}^{\mathrm{cond}}\right\}-2\langle\hat{x}\rangle \hat{\rho}^{\mathrm{cond}}\right) d W, \\
d \hat{y}=\frac{\alpha}{\hbar}\langle\hat{x}\rangle d t+d W / \sqrt{2},
\end{gathered}
$$

with a system Hamiltonian $H_{\text {sys }}$ and a coupling constant $\alpha$. Here the a posteriori state [56], which is often also called conditional quantum state [47-50,54], is defined as the projection of the joint system-device quantum state to the subspace in which the readout observable $\hat{y}$ has definite values of $\hat{y}\left(t^{\prime}\right)=y\left(t^{\prime}\right) \quad \forall 0<t^{\prime}<t$. The Wiener increment $d W$ describes a stochastic process that simultaneously drives the conditional quantum state and the measurement data, where both are stochastic processes. Different realizations of $d W$ correspond to different possible scenarios that could take place in reality. Note that $d W$ is in fact a differential of a real and not a complex Wiener noise.

In practice, it is not enough to only describe the stochastic process; we need to be able to obtain the conditional quan- tum state at any given time $t$ based on the system's initial quantum state $\hat{\rho}^{\text {cond }}(0)$ and measurement results $\left\{y\left(t^{\prime}\right), 0\right.$ $\left.<t^{\prime}<t\right\}$. This corresponds to a filtering problem in classical stochastic calculus. The probability distribution of any state variable $\hat{x}$ is simply the conditional probability

$$
P\left[\hat{x}(t) \mid\left\{y\left(t^{\prime}\right), 0<t^{\prime}<t\right\}\right],
$$

while the conditional expectation of $\hat{x}$ can be written as a functional over $\left\{y\left(t^{\prime}\right), 0<t^{\prime}<t\right\}$,

$$
x^{\mathrm{cond}}(t)=E\left[\hat{x}(t) \mid\left\{y\left(t^{\prime}\right), 0<t^{\prime}<t\right\}\right] .
$$

For Gaussian systems only expectations of quantities linear and quadratic in the variables are needed. The former can be obtained through a linear filter over $\hat{y}$, while the latter can be obtained by solving a time-domain Riccati equation. If the measurement process has started for sufficiently long timemuch longer than the time constant of transients- then the filters over $\hat{y}$ as well as the second-order moments are stationary. They can be obtained through the theory of Wiener filtering [53]. This will be the situation that we will consider in this paper.

For linear systems with Gaussian states a quantum filtering problem [57] can actually often be converted back into an essentially classical filtering problem. Let us consider the whole quantum-measurement process as divided into two parts [7]: a detector and a probe, where the latter is the measured system. Let $\hat{y}(t)$ be the Heisenberg operator of the detector's measurement output (e.g., a particular quadrature of the out-going optical field in an interferometric measurement) and $\hat{x}(t)$ any observable of the measured system (e.g., the position of a harmonic oscillator). Then we know that for a proper measurement device, (i) the principle of simultaneous measurability, i.e., the output of the measurement device $\hat{y}(t)$ at different times can be measured individually to arbitrary accuracy without imposing any fundamental limits, and (ii) the principle of causality, i.e., the measurement output at present does not respond to future changes in any system observable, will dictate that [51]

$$
\begin{gathered}
0=\left[\hat{y}(t), \hat{y}\left(t^{\prime}\right)\right] \quad \forall t, t^{\prime}, \\
0=\left[\hat{x}(t), \hat{y}\left(t^{\prime}\right)\right] \quad \forall t>t^{\prime},
\end{gathered}
$$

respectively. Note that $\hat{x}$ could be in fact any state variable such as momentum, position, or even the density operator of the probe. Then we could formally write that

$$
\hat{\rho}_{\left\{y\left(t^{\prime}\right), 0<t^{\prime}<t\right\}}^{\text {cond }}=\frac{\mathcal{P}_{\left[\hat{y}\left(t^{\prime}\right)=y\left(t^{\prime}\right), t^{\prime}<t\right]} \hat{\rho} \mathcal{P}_{\left[\hat{y}\left(t^{\prime}\right)=y\left(t^{\prime}\right), t^{\prime}<t\right]}}{\operatorname{tr}\left[\mathcal{P}_{\left[\hat{y}\left(t^{\prime}\right)=y\left(t^{\prime}\right), t^{\prime}<t\right]} \hat{\rho} \mathcal{P}_{\left[\hat{y}\left(t^{\prime}\right)=y\left(t^{\prime}\right), t^{\prime}<t\right]}\right]},
$$

where $\mathcal{P}$ projects onto the subspace on which the measurement operator takes the measured value. Henceforth the dependence of $\hat{\rho}^{\text {cond }}$ on $\left\{y\left(t^{\prime}\right), t^{\prime}<t\right\}$ will not be written explicitly, as in most of the literature.

Fortunately, Eqs. (9) and (10) also allow us to consider any filtering of the operator $\hat{y}\left(t^{\prime}\right)$ for $0<t^{\prime}<t$ as a classical process, i.e., treated with classical linear control theory, as long as the state of the system at $t$ is considered. In other words, system quantities, such as, e.g., $\hat{\rho}^{\text {cond }}(t)$, only depend on $\hat{y}\left(t^{\prime}\right)$ for $0<t^{\prime}<t$. Suppose we have observables $\hat{x}_{l}$ with 
$l=1,2, \ldots, n$ and proceed as in classical Wiener filtering. The key is to decompose the operator $\hat{x}_{l}$ into

$$
\hat{x}_{l}(t)=\int_{-\infty}^{t} d t^{\prime} K_{l}\left(t-t^{\prime}\right) \hat{y}\left(t^{\prime}\right)+\hat{R}_{l}(t)
$$

with

$$
\left\langle\hat{R}_{l}(t) \hat{y}\left(t^{\prime}\right)\right\rangle=\operatorname{tr}\left[\hat{\rho} \hat{R}_{l}(t) \hat{y}\left(t^{\prime}\right)\right]=0 .
$$

Furthermore, we will restrict ourselves to stable systems at later times when the initial states are no longer important. We further assume all linear observables to have zero unconditional expectation values. As shown in Appendix B, we then obtain the conditional first- and second-order moments as

$$
x_{l}^{\operatorname{cond}}(t)=\operatorname{tr}\left[\hat{\rho}^{\text {cond }} \hat{x}_{l}(t)\right]=\int_{-\infty}^{t} d t^{\prime} K_{l}\left(t-t^{\prime}\right) y\left(t^{\prime}\right)
$$

and

$$
\begin{aligned}
V_{x_{l} x_{m}}^{\mathrm{cond}} & \equiv \operatorname{tr}\left[\hat{\rho}^{\mathrm{cond}} \frac{\hat{x}_{l}(t) \hat{x}_{m}(t)+\hat{x}_{m}(t) \hat{x}_{l}(t)}{2}\right]-x_{l}^{\mathrm{cond}}(t) x_{m}^{\mathrm{cond}}(t) \\
& =\frac{1}{2}\left\langle\hat{R}_{l}(t) \hat{R}_{m}(t)+\hat{R}_{m}(t) \hat{R}_{l}(t)\right\rangle
\end{aligned}
$$

respectively. In this way, the conditional expectations of the linear variables are given as linear functionals of past measurement data $\left\{y\left(t^{\prime}\right), t^{\prime}<t\right\}$ and the conditional variances as steady-state constants.

Now we will recall how to obtain $K_{l}$ and $V_{x_{l} x_{m}}^{\text {cond }}$ in terms of unconditional correlation functions or cross spectra among system observables $\hat{x}_{l}$ and the output $\hat{y}$. Equation (13) leads to the Wiener-Hopf equation,

$$
C_{x_{l} y}\left(t-t^{\prime \prime}\right)-\int_{-\infty}^{t} d t^{\prime} K_{l}\left(t-t^{\prime}\right) C_{y y}\left(t^{\prime}-t^{\prime \prime}\right)=0 \quad \forall \quad t^{\prime \prime} \leq t .
$$

Here we have defined

$$
C_{a b}\left(t-t^{\prime}\right) \equiv\left\langle\hat{a}(t) \hat{b}\left(t^{\prime}\right)\right\rangle_{\mathrm{sym}} \equiv \operatorname{tr}\left[\hat{\rho} \frac{\hat{a}(t) \hat{b}\left(t^{\prime}\right)+\hat{b}\left(t^{\prime}\right) \hat{a}(t)}{2}\right],
$$

which stands for the symmetrized time-domain two-point correlation function between two arbitrary Heisenberg operators $\hat{a}(t)$ and $\hat{b}(t)$.

We have to suppose that $K_{l}(t)=0$ for $t<0$, i.e., making sure that the filter is a causal function, because due to Eq. (10) we are not allowed to use future measurement output for the classical filtering. Then we can rewrite Eq. (16) as

$$
C_{x_{l} y}(t)-\int_{-\infty}^{\infty} d t^{\prime} K_{l}\left(t^{\prime}\right) C_{y y}\left(t-t^{\prime}\right)=0 \quad \forall \quad t \geq 0 .
$$

In Fourier domain the condition in Eq. (18) is satisfied if the function

$$
L(\Omega)=S_{x_{l} y}(\Omega)-K_{l}(\Omega) S_{y y}(\Omega)
$$

is analytic in the lower-half complex plane while the Fourier transform of the filter function $K_{l}(\Omega)$ is analytic in the upperhalf complex plane. Furthermore, $L(\Omega)$ has to vanish at infinity because Eq. (18) must also be valid at $t=0$. These conditions uniquely define $K_{l}(\Omega)$. We have denoted with $S_{a b}(\Omega)$ the single-sided (cross-) spectral density among the operators $\hat{a}$ and $\hat{b}$ related to the two-point correlation as

$$
C_{a b}(t)=\frac{1}{2} \int_{-\infty}^{+\infty} \frac{d \Omega}{2 \pi} S_{a b}(\Omega) e^{-i \Omega t} .
$$

The Wiener-Hopf method provides the solution for $K_{l}(\Omega)$ as

$$
K_{l}(\Omega)=\frac{1}{s_{y}^{+}(\Omega)}\left[\frac{S_{x_{l} y}(\Omega)}{s_{y}^{-}(\Omega)}\right]_{+}
$$

where we have split $S_{y y}(\Omega)=s_{y}^{+}(\Omega) s_{y}^{-}(\Omega)$ in such a way that $s_{y}^{+}\left(s_{y}^{-}\right)$and its inverse are analytic functions in the upper-half (lower-half) complex plane. Because $S_{y y}(\Omega)$ is in general a rational function of $\Omega^{2}$ with real coefficients, we expect that $s_{y}^{+}(\Omega)=\left[s_{y}^{-}\left(\Omega^{*}\right)\right]^{*}$. In addition, $[F(\Omega) \cdots]_{+}$stands for taking the component of a function whose inverse Fourier transform has support only in positive times. Operationally, this could be obtained either by decomposing $F(\Omega)$ into

$$
F(\Omega)=\sum_{k} \frac{\alpha_{k}}{\Omega-\Omega_{k}}
$$

and only keeping terms whose $\Omega_{k}$ has negative imaginary parts or by inverse Fourier transforming $F(\Omega)$ into the time domain, eliminate the positive-time component, and then Fourier transform back. [Note that both approaches will become ambiguous when $F(\Omega)$ does not approach zero when $\Omega \rightarrow+\infty$.] Inserting Eq. (21) into Eqs. (12) and (15), we obtain

$$
V_{x_{l} x_{m}}^{\text {cond }}=\int_{0}^{\infty} \frac{d \Omega}{2 \pi} \operatorname{Re}\left(S_{x_{l} x_{m}}-\left[\frac{S_{x_{l} y}}{s_{y}^{-}}\right]_{+}\left[\frac{S_{x_{m} y}}{s_{y}^{-}}\right]_{+}^{*}\right) \text {. }
$$

A more transparent understanding of the filtering problem can be obtained when we formally define the causally whitened output as

$$
\hat{z}(\Omega) \equiv \frac{1}{s_{y}^{+}(\Omega)} \hat{y}(\Omega) .
$$

Because $1 / s_{y}^{+}$is analytic in the upper-half complex plane, $\hat{z}$ can be written as an integral over the history of $\hat{y}$. The random process $\hat{z}$ has a white spectrum. Moreover, we can write

$$
K_{l}(\Omega) \hat{y}(\Omega)=\left[S_{x_{l} z}(\Omega)\right]_{+} \hat{z}(\Omega) .
$$

In other words,

$$
x_{l}^{\text {cond }}(t)=\int_{-\infty}^{t} d t^{\prime} C_{x_{l}}\left(t-t^{\prime}\right) z\left(t^{\prime}\right)
$$

and 


$$
V_{x_{l} x_{m}}^{\text {cond }}=V_{x_{l} x_{m}}-\int_{-\infty}^{0} d t^{\prime} C_{x_{l} z}\left(-t^{\prime}\right) C_{x_{m^{z}}}\left(-t^{\prime}\right) .
$$

Clearly, Eqs. (26) and (27) are simply continuous versions of linear regression over a set of independent random variables.

In this paper with tools of Wiener filtering, we no longer need to write down SMEs. In fact, for realistic systems with multiple colored noise sources and non-Markovian dynamics, SMEs can only be obtained by increasing the dimension of the problem, which will definitely become cumbersome.

\section{MEASUREMENT CONFIGURATIONS WITH MARKOVIAN NOISE}

\section{A. General discussion}

Let us start our discussion generally by applying the technique from Sec. II to an abstract continuous linear Markovian measurement process, which monitors the center of mass position of a simple harmonic oscillator. Note that this analysis generalizes all previously obtained results using SMEs. We start with the linearized auxiliary Hamiltonian [51]

$$
H=H_{\mathrm{P}}+H_{\mathrm{D}}+\hat{x} \hat{F},
$$

where $H_{\mathrm{P}}$ describes the probe, i.e., the system which is measured, and $H_{\mathrm{D}}$ describes the measuring device. Here $\hat{x}$ is a linear observable of the probe and $\hat{F}$ is a detector variable. From Eq. (28) we can derive the following Langevin equations for the Heisenberg operators in the frequency domain:

$$
\begin{aligned}
& \hat{y}(\Omega)=\hat{Z}(\Omega)+\hat{x}(\Omega), \\
& \hat{x}(\Omega)=R_{x x}(\Omega) \hat{F}(\Omega),
\end{aligned}
$$

where in the time domain, the detector operator $\hat{y}$ and the probe operator $\hat{x}$ satisfy Eqs. (9) and (10). We suppose here that the two noise operators $\hat{Z}$ and $\hat{F}$ both have a white spectrum, which means that in the time domain, $\hat{Z}$ and $\hat{F}$ are white noise, with two-time correlation functions proportional to delta function. Such statistical characteristics make the measurement process a Markovian one-while this condition will be relaxed in Sec. V. Thus, we assume here that $\hat{Z}$ and $\hat{F}$ have the single-sided (cross-) spectral densities [cf. Eq. (20)] $S_{Z Z} \geq 0, S_{F F} \geq 0$, and $S_{Z F} \in \mathrm{R}$, which satisfy the Heisenberg relation of the measurement process [7]

$$
S_{Z Z} S_{F F}-S_{Z F}^{2}=\mu \hbar^{2}, \quad \mu \geq 1,
$$

which arises from the requirement that the Heisenberg output operators at different times must commute [cf. Eq. (9)] and guarantees that the level of back action is just enough to enforce the Heisenberg uncertainty relation of the test mass [7]. In the case of Gaussian noise only, we have $\mu=1$ if and only if the measurement process is purely quantum.

The linear response function of a damped harmonic oscillator is given by

$$
R_{x x}(\Omega)=-\frac{1}{m\left(\Omega^{2}+i \gamma_{m} \Omega-\omega_{m}^{2}\right)},
$$

with the eigenfrequency $\omega_{m}$, the damping rate $\gamma_{m} \ll \omega_{m}$, and the mass $m$. Then we can easily assemble the single-sided spectral densities [cf. Eq. (20)] of the measurement process, using Eq. (29)-(32), as

$$
\begin{aligned}
S_{y y}(\Omega)= & \left\langle y(\Omega) y^{\dagger}(\Omega)\right\rangle_{\mathrm{sym}}=S_{Z Z}+2 \operatorname{Re}\left\{R_{x x}(\Omega)\right\} S_{Z F} \\
& +\left|R_{x x}(\Omega)\right|^{2} S_{F F}=m^{2}\left|R_{x x}(\Omega)\right|^{2}\left[\Omega^{4}-\Omega^{2}\left(2 q_{1}-\gamma_{m}^{2}\right)\right. \\
& \left.+q_{2}^{2}\right] S_{Z Z}, \\
S_{x y}(\Omega)= & \left\langle x(\Omega) y^{\dagger}(\Omega)\right\rangle_{\mathrm{sym}}=-m\left|R_{x x}(\Omega)\right|^{2}\left(\Omega^{2}-i \gamma_{m} \Omega-\omega_{m}^{2}\right. \\
& \left.-\frac{S_{F F}}{S_{Z F}}\right) S_{Z F}, \\
& S_{x x}(\Omega)=\left\langle x(\Omega) x^{\dagger}(\Omega)\right\rangle_{\text {sym }}=\left|R_{x x}(\Omega)\right|^{2} S_{F F},
\end{aligned}
$$

where the subscript sym stands again for symmetrization and we have defined the coefficients

$$
\begin{gathered}
q_{1} \equiv \omega_{m}^{2}+\frac{S_{Z F}}{m S_{Z Z}}, \\
q_{2} \equiv \sqrt{\omega_{m}^{4}+2 \omega_{m}^{2} \frac{S_{Z F}}{m S_{Z Z}}+\frac{S_{F F}}{m^{2} S_{Z Z}}} .
\end{gathered}
$$

Both $q_{1,2}$ have the dimension of frequency squared, while $q_{2}$ is always positive. We also note that $\left|q_{1}\right| \leq q_{2}$.

Note that from Eq. (33) we can recover a quantum limit of the measurement process: if we have $S_{Z F}=0$, the spectral density of the measurement noise satisfies

$$
S_{y y}(\Omega) \geq 2 \sqrt{S_{Z Z} S_{F F}}\left|R_{x x}(\Omega)\right| \geq 2 \sqrt{\mu} \hbar\left|R_{x x}(\Omega)\right|=\sqrt{\mu} S_{\mathrm{SQL}}(\Omega) .
$$

For a free mass we can then recover Eq. (2) from Eq. (38). Note that for the free mass the first inequality sign in Eq. (38) becomes an equality sign at $\Omega=\Omega_{q}$, where the measurement frequency is defined by

$$
\Omega_{q}^{2} \equiv \sqrt{\frac{S_{F F}}{m^{2} S_{Z Z}}} .
$$

Therefore, the measurement frequency $\Omega_{q}$ is the frequency at which the noise spectral density of a Markovian measurement process with $S_{Z F}=0$ approaches most its free-mass SQL.

Now it is straightforward to derive the conditional variances assuming $\hat{p}=-i m \Omega \hat{x}$ and using the Wiener filtering method from Sec. II. For this it is actually crucial to spectral factorize $S_{y y}(\Omega)$ in Eq. (33) into

$$
\begin{gathered}
s_{y}^{+}(\Omega)=\sqrt{S_{Z Z}} \frac{\left(\Omega-\Omega_{1}\right)\left(\Omega-\Omega_{2}\right)}{\Omega^{2}+i \gamma_{m} \Omega-\omega_{m}^{2}}, \\
s_{y}^{-}(\Omega)=\left[s_{y}^{+}(\Omega)\right]^{*},
\end{gathered}
$$

where 


$$
\Omega_{1,2}=\left( \pm \sqrt{q_{2}+q_{1}}-i \sqrt{q_{2}-q_{1}}\right) / \sqrt{2} .
$$

Inserting Eqs. (34) and (35) as well as $S_{p y}(\Omega)=$ $-i m \Omega S_{x y}(\Omega), S_{p p}(\Omega)=m^{2} \Omega^{2} S_{x x}(\Omega)$, and $S_{x p}(\Omega)=i m \Omega S_{x x}(\Omega)$ together with Eq. (41) into Eq. (23), the conditional steadystate covariance matrix can be put in the perfect oscillator limit, i.e., with $\gamma_{m} \rightarrow 0$, into the following form:

$$
\mathbf{V}=\sqrt{\mu} \mathbf{D}\left(\begin{array}{cc}
\sqrt{\frac{2 q_{2}}{q_{1}+q_{2}}} & \sqrt{\frac{q_{2}-q_{1}}{q_{2}+q_{1}}} \\
\sqrt{\frac{q_{2}-q_{1}}{q_{2}+q_{1}}} & \sqrt{\frac{2 q_{2}}{q_{1}+q_{2}}}
\end{array}\right) \mathbf{D}^{T},
$$

where we have defined

$$
\mathbf{D}=\left(\begin{array}{cc}
\sqrt{\frac{\hbar}{2 m \sqrt{q_{2}}}} & 0 \\
0 & \sqrt{\frac{\hbar m \sqrt{q_{2}}}{2}}
\end{array}\right)
$$

and $q_{1,2}$ are given in Eqs. (36) and (37). Equation (43) gives us the most general covariance matrix of the conditional Gaussian state of a lossless harmonic oscillator under any linear Markovian position measurement. In the covariance matrix $\mathbf{V}$, the matrix $\mathbf{D}$ sets the scale of the quantum fluctuations to be comparable to those of the vacuum state of a harmonic oscillator with eigenfrequency $\sqrt{q_{2}}$. Depending on the ratio $q_{1} / q_{2}$, which always lies between -1 and +1 , the noise ellipse of the vacuum state is deformed into one where position and momentum are correlated (unless if $q_{1} / q_{2} \approx 1$ ) while the area is conserved. This corresponds to a unitary transformation among Gaussian states. Then $\mu$ finally enlarges the noise ellipse with a uniform factor, converting the pure state into a mixed state (unless if $\mu=1$ ). We have found that the conditional variances completely coincide with those obtained from SMEs. With Eq. (43) we can for instance easily reproduce Eqs. (2.8a)-(2.8c) from Ref. [48].

A Gaussian state is pure if and only if its uncertainty product is Heisenberg limited. Therefore, it makes sense to quantify the mixedness of the conditional state by its uncertainty product, here given by

$$
\operatorname{det} \mathbf{V}=V_{x x} V_{p p}-V_{x p}^{2}=\mu \frac{\hbar^{2}}{4},
$$

which is identical to the uncertainty product of the measurement process. This simply shows that in the Markovian case, any measurement will produce a pure conditional state of a lossless harmonic oscillator if and only if it is a quantum measurement. Moreover, the uncertainty product is even independent of the system's mechanical properties such as the oscillator's mass and eigenfrequency. Note that in Appendix A we have introduced how the uncertainty product is related to an effective occupation number $\mathcal{N}_{\text {eff }}$. It turns out, when using Eq. (A4), that here the effective eigenfrequency realizing the effective occupation number is given by $\omega_{\text {eff }}=\sqrt{q_{2}}$.

The covariance matrix in Eq. (43) becomes obviously diagonal and the correlation between $\hat{x}$ and $\hat{p}$ in the conditional state vanishes if and only if $q_{1}=q_{2}$. But this is strictly for- bidden due to the Heisenberg uncertainty principle. However, in a certain limit they can become very close [41]. With a higher difference in $q_{1}$ and $q_{2}$ not only the correlation in $\hat{x}$ and $\hat{p}$ but also the uncertainty product $\mu$ increase.

In order to obtain the conditional state, as given in Eq. (43), the measurement data have to be filtered in real time using the Wiener filter functions for position and momentum which are given in the frequency domain by

$$
\begin{aligned}
& K_{x}(\Omega)=\sqrt{2} i \sqrt{q_{2}-q_{1}} \frac{\Omega-\Omega_{3}}{\left(\Omega-\Omega_{1}\right)\left(\Omega-\Omega_{2}\right)}, \\
& K_{p}(\Omega)=i m\left(q_{2}-\omega_{m}^{2}\right) \frac{\Omega+\omega_{m}^{2} / \Omega_{3}}{\left(\Omega-\Omega_{1}\right)\left(\Omega-\Omega_{2}\right)},
\end{aligned}
$$

where $\Omega_{3}=i / \sqrt{2}\left(\omega_{m}^{2}-q_{2}\right) / \sqrt{q_{2}-q_{1}}$. Note that the poles of the Wiener filter $\Omega_{1,2}$ are actually equal to the zeros of the measurement's output spectrum $S_{y y}(\Omega)$ as given in Eq. (42), which in turn correspond to the frequencies of maximal sensitivity and are therefore easy to find.

Let us now have a closer look on the noise model we will use throughout this section. We will categorize the noise into two groups: (i) the one which is a result of the measurement process itself will be denoted by quantum noise and (ii) the additional noise will be called classical noise which does not directly arise from the measurement process, usually has no correlation in $\hat{Z}$, and $\hat{F}$ does not have to satisfy Eq. (31). Then the two noise sources combine as $S_{F F}=S_{F F}^{q}+S_{F F}^{\mathrm{cl}}$ and $S_{Z Z}=S_{Z Z}^{q}+S_{Z Z}^{\mathrm{cl}}$.

The quantum noise is dominated at high frequencies by shot noise which is covered by $S_{Z Z}^{q}$ and at low frequencies by back-action noise which is covered by $S_{F F}^{q}$. The latter one is represented by the radiation-pressure noise in the case of a measurement with light. If both are uncorrelated, i.e., $S_{Z F}^{q}$ $=0$, they result in the SQL. Then the quantum-noise spectral density is limited from below by the free-mass SQL as shown in Eq. (38) and also in Fig. 2. The quantum noise touches the free-mass SQL at the frequency $\Omega=\Omega_{q}$.

The other noise source can also be divided into two parts: a classical force noise $S_{F F}^{\mathrm{cl}}$ is added to $S_{F F}$ which acts directly on the center of mass of the measured object. An example is the noise corresponding to the velocity damping of a harmonic oscillator, i.e., its Brownian motion [50] in the Markovian approximation. While in real interferometric experiments the classical force noise is more generally due to for instance seismic noise or thermal noise in the suspension of the mirrors. The classical sensing noise $S_{Z Z}^{\mathrm{cl}}$ is only a pseudomotion of the measured object and may be due to the following reasons: (i) on the one hand due to thermal fluctuations of the mirror's shape as for example mirror internal thermal noise which makes only the mirror surface move with respect to its center of mass; (ii) on the other hand be due to optical losses; or (iii) due to photodetection inefficiency. Therefore, our sensing noise is somehow generalized from what is conventionally understood when using the term sensing noise. Note that for detecting GWs, only the total noise matters, yet for studying quantum-state preparation, it is important to make distinctions between sensing and force noises and between quantum and classical noises. Through- 


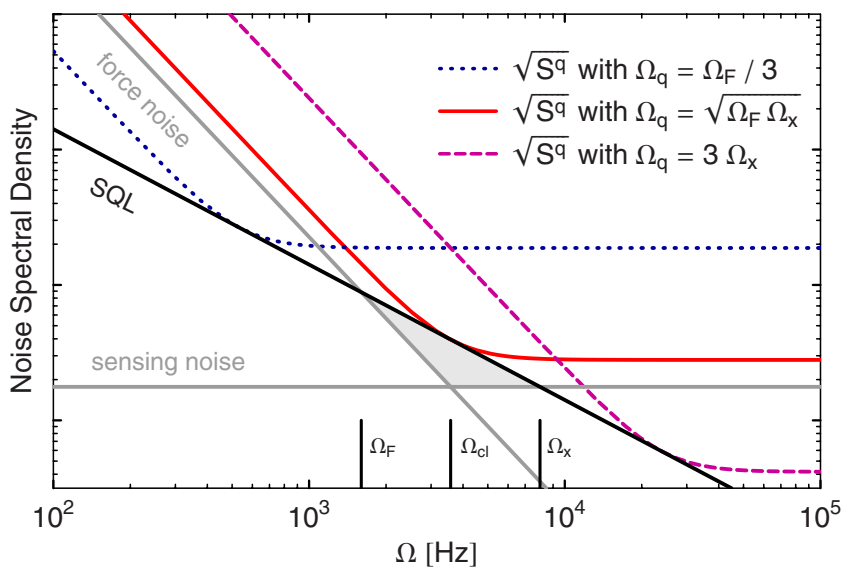

FIG. 2. (Color online) Example noise spectral densities (in arbitrary units) of a Markovian measurement process observing a free mass: the quantum-noise spectral density at different values of the measurement frequency $\Omega_{q}$ as well as the spectral densities corresponding to a classical force noise and a sensing noise (marked in the plot). The gray-shadowed region marks the classical-noise SQL beating which we have chosen to have $\Omega_{x} / \Omega_{F}=5$.

out this section we will assume both classical-noise sources to have a white spectrum. Then they can be characterized by the frequencies $\Omega_{F}$ for the force noise and $\Omega_{x}$ for the sensing noise at which their noise spectral density intersects with the free-mass SQL (cf. Fig. 2). These classical-noise frequencies are defined by the following relations:

$$
\begin{gathered}
S_{F F}^{\mathrm{cl}}=2 \hbar m \Omega_{F}^{2}, \\
S_{Z Z}^{\mathrm{cl}}=\frac{2 \hbar}{m \Omega_{x}^{2}} .
\end{gathered}
$$

Note that the classical force noise together with the classical sensing noise can open a window in which both are below the free-mass SQL as indicated by the gray-shadowed region in Fig. 2. The total classical noise is then even completely below the SQL in a certain frequency band if and only if the classical-noise sources satisfy

$$
S_{F F}^{\mathrm{cl}} S_{Z Z}^{\mathrm{cl}}<\hbar^{2} \Leftrightarrow \Omega_{x} / \Omega_{F}>2,
$$

which turns into a constraint for the classical-noise frequency ratio. If Eq. (50) holds, the classical noise is equal to a minimum of $\left(2 \Omega_{F} / \Omega_{x}\right)$ times the free-mass SQL at the frequency $\Omega=\Omega_{\mathrm{cl}} \equiv \sqrt{\Omega_{F} \Omega_{x}}$ which reads

$$
\min _{\Omega}\left\{\frac{S_{\mathrm{cl}}(\Omega)}{S_{\mathrm{SQL}}(\Omega)}\right\}=\frac{S_{\mathrm{cl}}\left(\Omega_{\mathrm{cl}}\right)}{S_{\mathrm{SQL}}\left(\Omega_{\mathrm{cl}}\right)}=\frac{2 \Omega_{F}}{\Omega_{x}} .
$$

Since here the classical noise has the largest separation to the SQL, we can understand the factor $\left(2 \Omega_{F} / \Omega_{x}\right)$ as the classical-noise-SQL-beating factor.

\section{B. Very low finesse cavity and free-mass scenario with vacuum input}

Let us specify our general discussion from Sec. III A by considering the simple situation of a laser beam incident on a suspended (with a high quality factor and a low eigenfrequency, i.e., with $\left.\gamma_{m} \ll \omega_{m} \ll 1\right)$ mirror, where the output field is monitored by a perfect balanced homodyne detection at a frequency-independent angle $\zeta$. This corresponds to a mirror in a cavity with infinitely large bandwidth (or at least much larger than $\Omega_{q}$, as we shall quantify in Sec. V A), in which case the dynamics of the cavity mode can be ignored or adiabatically eliminated. Note that the following analysis is also valid for the dark port fields entering and leaving an equal-arm Michelson interferometer with movable end mirrors and the differential motion between these mirrors-but then the mirror mass $m$ in the following discussion has to be substituted by the reduced mass $m / 2$. Then the quantummeasurement process can be described by the following spectral densities [54]:

$$
\begin{gathered}
S_{Z Z}=\frac{\hbar^{2}}{\alpha^{2}} \tan ^{2} \zeta+\frac{\hbar^{2}}{\alpha^{2}}+\frac{2 \hbar}{m \Omega_{x}^{2}}, \\
S_{F F}=\alpha^{2}+2 m \hbar \Omega_{F}^{2}, \\
S_{Z F}=\hbar \tan \zeta,
\end{gathered}
$$

where the coupling constant is defined by

$$
\alpha=\sqrt{\frac{8 P \omega_{0} \hbar}{c^{2}}} .
$$

Here $P$ is the circulating laser power and $\omega_{0}$ is the laser angular frequency.

In the free-mass limit, i.e., with $\omega_{m} \rightarrow 0$, the conditional variances from Eq. (43) simplify to

$$
\begin{aligned}
V_{x x}= & \frac{\hbar}{\sqrt{2} m \Omega_{q}} \sqrt{1+\tan ^{2} \zeta+2 \xi_{x}^{2}} \\
& \times\left[\sqrt{\left(1+2 \xi_{F}^{2}\right)\left(1+\tan ^{2} \zeta+2 \xi_{x}^{2}\right)}-\tan \zeta\right]^{1 / 2}, \\
V_{p p}= & \frac{\hbar m \Omega_{q}}{\sqrt{2}} \sqrt{1+2 \xi_{F}^{2}} \times\left[\sqrt{\left(1+2 \xi_{F}^{2}\right)\left(1+\tan ^{2} \zeta+2 \xi_{x}^{2}\right)}\right. \\
- & \tan \zeta]^{1 / 2}, \\
V_{x p}= & \frac{\hbar}{2}\left[\sqrt{\left(1+2 \xi_{F}^{2}\right)\left(1+\tan ^{2} \zeta+2 \xi_{x}^{2}\right)}-\tan \zeta\right],
\end{aligned}
$$

while the uncertainty product of the state is given by

$$
\begin{aligned}
\operatorname{det} \mathbf{V}= & \frac{\hbar^{2}}{4}\left[\left(1+2 \xi_{F}^{2}\right)\left(1+2 \xi_{x}^{2}\right)+2 \xi_{F}^{2} \tan ^{2} \zeta\right] \geq \frac{\hbar^{2}}{4}(1 \\
& \left.+2 \xi_{F} \xi_{x}\right)^{2}
\end{aligned}
$$

Here we have defined the two ratios, $\xi_{F} \equiv \Omega_{F} / \Omega_{q}$ and $\xi_{x}$ $\equiv \Omega_{q} / \Omega_{x}$ with $\Omega_{q}=\alpha / \sqrt{\hbar m}$. Then we can recover Eq. (4) and with the formula derived in Appendix A we obtain

$$
\mathcal{N}_{\text {eff }}=\frac{S_{\mathrm{cl}}\left(\Omega_{\mathrm{cl}}\right)}{2 S_{\mathrm{SQL}}\left(\Omega_{\mathrm{cl}}\right)} .
$$

In the quantum-noise limit with $\xi_{F}=\xi_{x}=0$ the conditional state is pure for any measurement frequency $\Omega_{q}$ and homo- 
dyne detection angle $\zeta$. This defines the conditional ground state of a free mass,

$$
\begin{gathered}
V_{x x}=\frac{\hbar}{\sqrt{2} m \Omega_{q}}, \\
V_{p p}=\frac{\hbar m \Omega_{q}}{\sqrt{2}}, \\
V_{x p}=\frac{\hbar}{2} .
\end{gathered}
$$

In this quantum-noise limit the Wiener filter functions for position and momentum become equal to simple decaying cosine functions at the measurement frequency and read

$$
\begin{gathered}
K_{x}(t)=\sqrt{2} \Omega_{q} e^{-\Omega_{q} t / \sqrt{2}} \cos \left(\frac{\Omega_{q} t}{\sqrt{2}}\right), \\
K_{p}(t)=\sqrt{2} m \Omega_{q}^{2} e^{-\Omega_{q} t / \sqrt{2}} \cos \left(\frac{\Omega_{q} t}{\sqrt{2}}+\frac{\pi}{4}\right) .
\end{gathered}
$$

Here it becomes apparent that the inverse of the measurement frequency $\Omega_{q}$ (at which the total noise approaches SQL the most) is also the time scale at which information regarding test-mass position and momentum has to be extracted from the output data.

In the presence of classical noise it is clear from Eq. (59) that one should measure the phase quadrature, i.e., $\zeta=0$, in order to minimize the uncertainty product. That means that in order to obtain a small uncertainty product, it is not required to remove the quantum back-action noise from the output, as one would benefit from when trying to detect GWs [9]-in the contrary, for that purpose it would be even destructive to do so. This is understandable since here the aim is to learn as much as possible about the mirror motion, and the effect of quantum back action is an important content of the mirror motion. For example, suppose we chose to measure a backaction-evading observable using an oscillator with negligible classical noise. In this case, we would have an output channel that has a minimal power spectrum and is hence ideal for measuring any classical force that acts onto the mirror. Fluctuation in the mirror motion and the momentum driven by back action, on the other hand, would almost diverge around the resonant frequency. The output field, containing absolutely no information about the back action, would not be able to remove this fluctuation via conditioning. This would then result in a conditional state equal to the unconditional state with very large variances.

We learn from Eqs. (56)-(58) that the effect of the classical force noise in the conditional variances is suppressed with a higher measurement frequency (random force has less time to act and accumulate) while the classical sensing noise is suppressed with a lower measurement frequency (random sensing noise has longer time to average out).

Moreover, in the absence of any classical sensing noise the test-mass state becomes pure with an infinitely strong measurement (in which $\Omega_{q}$ approaches $+\infty$ ): all classical forces acting on the test mass can be neglected in presence of the strong back-action force and the test mass reaches the conditional ground state at the infinite measurement frequency. Vice versa, in the theoretical absence of classical force noise, the test-mass state becomes pure in the limit of an infinitely weak measurement (in which $\Omega_{q}$ approaches 0 ): if the test-mass motion is only driven by the measurement's back action but this motion is then unfortunately hidden in the measurement output because it is covered by the classical sensing noise, the best idea would be not to measure the test mass at all. If both classical-noise sources are present, the uncertainty product is minimized further with an optimal power which accomplishes a balancing between classical force and sensing noise, i.e., with $\xi_{F}=\xi_{x}$. This produces an equal sign in the second line of Eq. (59) and is true for a measurement frequency of $\Omega_{q}=\Omega_{\mathrm{cl}}$. This simply means that the quantum noise should touch the free-mass SQL at the frequency where the classical noise has the maximal separation to that limit (cf. Fig. 2). The expression of the minimal uncertainty product and the one of the minimal effective occupation numbers are then functions of the classical-noiseSQL-beating factor.

For a mechanical object there is not a fundamental definition of which state is vacuum since it is possible to vary the potential well it lies in. Nevertheless, states with nonzero correlation among displacement and momentum can always be regarded as squeezed, and we can often discuss whether one state is more position squeezed (momentum antisqueezed) or more position antisqueezed (momentum squeezed) compared to another. We have illustrated the squeezing situation by plotting noise ellipses obtained with various homodyne detections (see upper panel of Fig. 3). As the homodyne detection angle $\zeta$ varies from optimal value 0 to $-\pi / 2$, the semimajor axis of the noise ellipse, i.e., the antisqueezed quadrature, becomes rotated into the direction of the position. At the same time the ellipse becomes more stretched, i.e., the squeezed quadrature is more squeezed, while the antisqueezed quadrature is more antisqueezed. As $\zeta$ varies from 0 to $\pi / 2$, the semimajor axis of the noise ellipse also rotates into the direction of the position but the ellipse becomes rather bulged. Furthermore, we show how the noise ellipse changes as the measurement frequency is not chosen to be equal to the geometrical mean of the two classicalnoise frequencies, i.e., $\Omega_{q} \neq \Omega_{\mathrm{cl}}$. For phase quadrature detection as in the lower panel of Fig. 3, a slow measurement, i.e., with a low measurement frequency $\Omega_{q}<\Omega_{\mathrm{cl}}$, generates a position antisqueezed (and momentum squeezed) conditional state, while a fast measurement with $\Omega_{q}>\Omega_{\mathrm{cl}}$ generates a position squeezed (and momentum antisqueezed) conditional state. In addition, the deviation from the optimal measurement frequency, i.e., $\Omega_{q} \neq \Omega_{\mathrm{cl}}$, always produces a less pure states.

\section{Very low finesse cavity and free-mass scenario with squeezed vacuum input}

In Sec. III C we have only treated in-going coherent vacuum states but one could also think about squeezed vacuum states coupling to the mirror [9]. This corresponds to inserting squeezed states into an interferometer's dark port. 

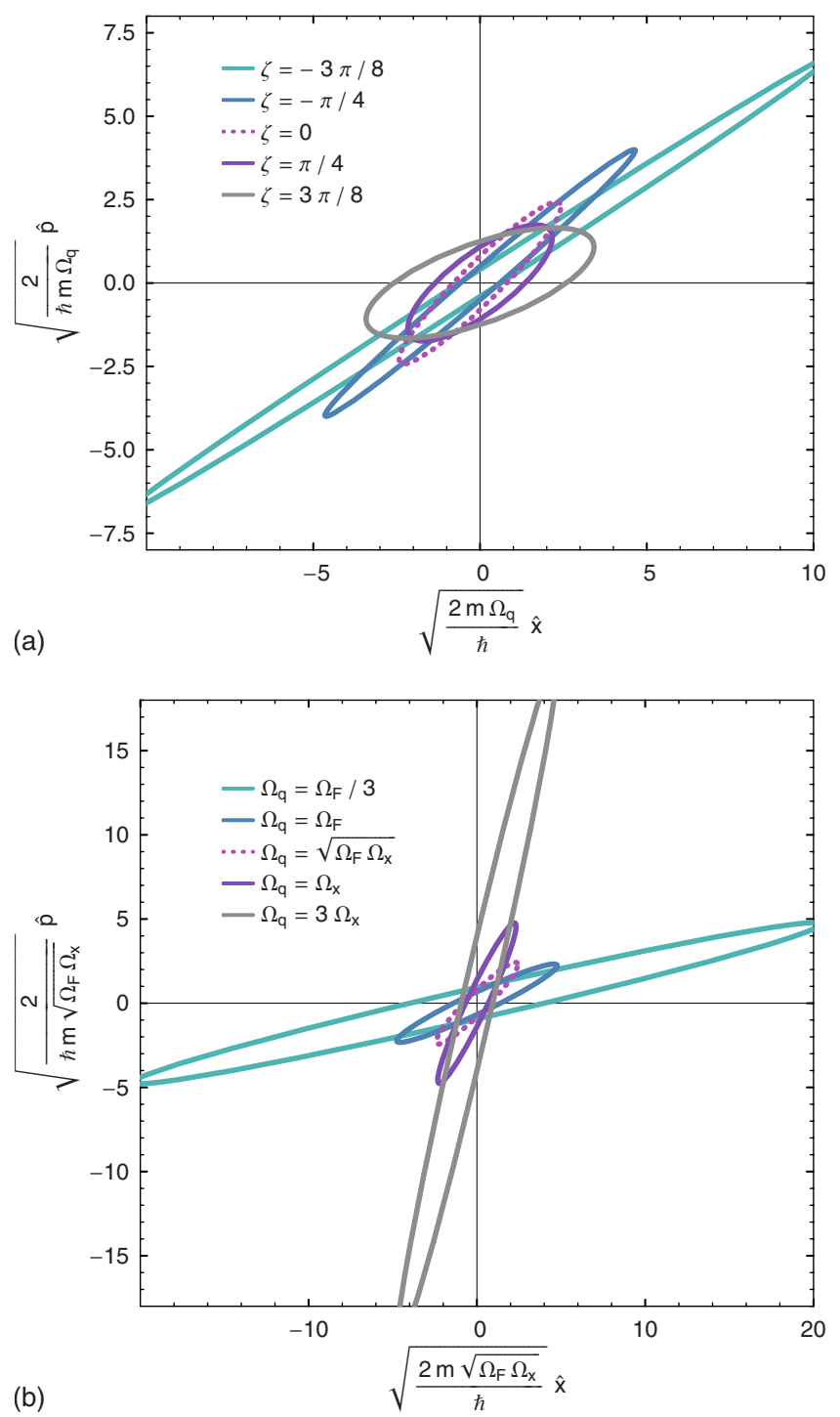

FIG. 3. (Color online) Test-mass squeezing normalized with respect to the conditional ground state of a free mass for (upper panel) optimal measurement frequency $\Omega_{q}=\Omega_{\mathrm{cl}} \equiv \sqrt{\Omega_{F} \Omega_{x}}$ and different homodyne detection angles (increasing from bright to dark color) and (lower panel) for different measurement frequencies (increasing from bright to dark color) at phase quadrature detection including in both cases a certain classical-noise budget: we have chosen $\Omega_{x} / \Omega_{F}=5$.

By doing so the quantum limited sensitivity of an interferometer can be enhanced [58]. The free-mass conditional state is given by

$$
\begin{aligned}
V_{x x}= & \frac{\hbar}{\sqrt{2} m \Omega_{q}} \sqrt{\lambda_{-}^{2}+2 \xi_{x}^{2}} \times\left[\sqrt{\left(\lambda_{+}^{2}+2 \xi_{F}^{2}\right)\left(\lambda_{-}^{2}+2 \xi_{x}^{2}\right)}\right. \\
& \left.-\sin 2 \varphi_{\mathrm{op}} \sinh 2 r_{\mathrm{op}}\right]^{1 / 2} \\
V_{p p}= & \frac{\hbar m \Omega_{q}}{\sqrt{2}} \sqrt{\lambda_{+}^{2}+2 \xi_{F}^{2}} \times\left[\sqrt{\left(\lambda_{+}^{2}+2 \xi_{F}^{2}\right)\left(\lambda_{-}^{2}+2 \xi_{x}^{2}\right)}\right. \\
& \left.-\sin 2 \varphi_{\mathrm{op}} \sinh 2 r_{\mathrm{op}}\right]^{1 / 2},
\end{aligned}
$$

$$
V_{x p}=\frac{\hbar}{2}\left[\sqrt{\left(\lambda_{+}^{2}+2 \xi_{F}^{2}\right)\left(\lambda_{-}^{2}+2 \xi_{x}^{2}\right)}-\sin 2 \varphi_{\mathrm{op}} \sinh 2 r_{\mathrm{op}}\right] .
$$

Here we have defined $\lambda_{ \pm}^{2}=\cosh 2 r_{\mathrm{op}} \pm \cos 2 \varphi_{\mathrm{op}} \sinh 2 r_{\mathrm{op}}$, where $(20 / \ln 10) r_{\text {op }}>0$ gives the optical squeezing strength in $\mathrm{dB}$ at a squeezing angle of $\varphi_{\mathrm{op}}$. Then the purity of the conditional state can be inferred from

$$
\begin{aligned}
\operatorname{det} \mathbf{V}= & \frac{\hbar^{2}}{4}\left[\left(\lambda_{+}^{2}+2 \xi_{F}^{2}\right)\left(\lambda_{-}^{2}+2 \xi_{x}^{2}\right)-\sin ^{2} 2 \varphi_{\mathrm{op}} \sinh ^{2} 2 r_{\mathrm{op}}\right] \\
\geq & \frac{\hbar^{2}}{4}\left[1+4 \xi_{F}^{2} \xi_{x}^{2}+2\left(\xi_{F}^{2}+\xi_{x}^{2}\right) \cosh 2 r_{\mathrm{op}}-2 \mid \xi_{F}^{2}\right. \\
& \left.-\xi_{x}^{2} \mid \sinh 2 r_{\mathrm{op}}\right] \geq \frac{\hbar^{2}}{4}\left(1+2 \xi_{F} \xi_{x}\right)^{2} .
\end{aligned}
$$

The equality of the first inequality sign in Eq. (69) is achieved at $\varphi_{\text {op }}=0$ for $\xi_{F}^{2}>\xi_{x}^{2}$ and at $\varphi_{\text {op }}=\pi / 2$ for $\xi_{F}^{2}<\xi_{x}^{2}$, i.e., by squeezing either the phase or the amplitude quadrature, respectively. Note that in Eq. (69) for any $\Omega_{q}$, the same minimum as in Eq. (59) is reached if $r_{\mathrm{op}}=\operatorname{arctanh}\left[\mid \xi_{F}^{2}\right.$ $\left.-\xi_{x}^{2} \mid /\left(\xi_{F}^{2}+\xi_{x}^{2}\right)\right] / 2$-even when having $\xi_{F} \neq \xi_{x}$. Therefore, even with input squeezing, the conditional state cannot become more pure than with coherent input, but the demands on the required measurement frequency and with this the constraints on the optical power, which is needed in order to obtain a certain uncertainty product, can be relaxed. In real experiments the optical power is of course always limited and squeezed input becomes a very important tool.

It has turned out that the conditional variances are in principle even analytically equivalent in the following two cases: (i) input squeezing at a flexible but frequency-independent angle or (ii) flexible amount of available optical power and a flexible but frequency-independent homodyne detection angle. This can easily be seen by replacing the homodyne detection angle $\tan \zeta \rightarrow \sin 2 \varphi_{\text {op }} \sinh 2 r_{\text {op }}$ and the measurement frequency $\Omega_{q} \rightarrow \lambda_{+} \Omega_{q}$ in Eqs. (56)-(58). Then we simply end up with Eqs. (66)-(68). Here we can directly see that using input squeezing allows to change the parameters within $\Omega_{q}$ such as the optical power, the laser frequency, and the mirror mass but by modifying the input squeezing parameter $\lambda_{+}$we can at the same time maintain the measurement frequency.

Even though a homodyne detection different from the phase quadrature and input squeezing do both not help with increasing the purity of the conditional state they increase the squeezing of the conditional test-mass state. Furthermore, with a certain homodyne detection angle or with a certain input squeezing it is possible to minimize the position and momentum correlation in the conditional state.

\section{MACROSCOPIC ENTANGLEMENT}

The concept of entangled states is one of the most important phenomena when entering the quantum regime. In Sec. $\mathrm{V}$ A we will see that the entanglement between the cavity mode and the mirror motion is in fact responsible for a degeneration of the purity of the mirror's quantum state. In this 
section we will explain how we can prepare quantum entanglement in position and momentum between the centers of mass of the two end mirrors in the north $\hat{x}^{\mathrm{n}}$ and the east $\hat{x}^{\mathrm{e}}$ arm of a simple but power-recycled Michelson interferometer using the conditional states as derived in Sec. III B. The end mirrors are suspended as pendulums but with a very low eigenfrequency. Therefore, such an experiment would be in direct analogy to the Einstein-Podolsky-Rosen gedanken experiment [59]. Here we will basically extend the discussion carried out in Ref. [54].

Recall that the common $\left(\hat{x}^{\mathrm{e}}+\hat{x}^{\mathrm{n}}\right)$ and the differential $\left(\hat{x}^{\mathrm{e}}\right.$ $\left.-\hat{x}^{n}\right)$ modes of motion between the two end mirrors are independent and can each be sensed by a homodyne detection at the bright and the dark ports, respectively, as suggested in Ref. [54]. As already mentioned before, if using the reduced mass of the mirrors, the conditional variances as derived in Sec. III B hold for the differential mode observed at the dark port of the interferometer. But in order to describe the common mode they have to be slightly modified since the powerrecycling cavity-here with high bandwidth and therefore an adiabatically eliminated cavity mode-with transmissivity $\tau$ enhances the measurement strength $\alpha^{\mathrm{c}}=2 / \tau \alpha>\alpha$ and is therefore different to the one associated with the differential mode $\alpha^{\mathrm{d}}=\alpha$. Furthermore, the common mode will suffer additionally to the classical force noise and the classical sensing noise-note that we suppose that these two classicalnoise sources are equally distributed into common and differential modes-from laser noise since the in-going modulation fields at the bright port are usually not in vacuum states. We have to make the following additional replacements in Eqs. (56)-(58) in order to obtain the conditional variances of the common mode: $\left(1+2 \xi_{F}^{2}\right) \rightarrow\left(S_{a_{1} a_{1}}+2 \xi_{F}^{2}\right)$ and $\left(1+\tan ^{2} \zeta+2 \xi_{x}^{2}\right) \rightarrow\left(S_{a_{2} a_{2}}+S_{a_{1} a_{1}} \tan ^{2} \zeta+2 \xi_{x}^{2}\right)$ as well as replacing the detached $(-\tan \zeta)$ in each variance by ( $-S_{a_{1} a_{1}} \tan \zeta$ ). Here $S_{a_{1} a_{1}}, S_{a_{2} a_{2}} \geq 1$ are the (frequencyindependent) spectra of the technical laser noise in amplitude and phase, respectively.

Then we can assemble the conditional state of the entire system: the combined covariance among $\left(x^{\mathrm{e}}, p^{\mathrm{e}}, x^{\mathrm{n}}, p^{\mathrm{n}}\right)$ simply reads

$$
\mathbf{V}_{\text {tot }}=\left(\begin{array}{cc}
\mathbf{V}_{\text {ee }} & \mathbf{V}_{\text {en }} \\
\mathbf{V}_{\text {ne }} & \mathbf{V}_{\mathrm{nn}}
\end{array}\right)
$$

with

$$
\begin{aligned}
& \mathbf{V}_{\mathrm{nn}}=\mathbf{V}_{\mathrm{ee}}=\left(\begin{array}{lc}
\left(V_{x x}^{\mathrm{c}}+V_{x x}^{\mathrm{d}}\right) / 4 & \left(V_{x p}^{\mathrm{c}}+V_{x p}^{\mathrm{d}}\right) / 2 \\
\left(V_{x p}^{\mathrm{c}}+V_{x p}^{\mathrm{d}}\right) / 2 & V_{p p}^{\mathrm{c}}+V_{p p}^{\mathrm{d}}
\end{array}\right), \\
& \mathbf{V}_{\mathrm{en}}=\mathbf{V}_{\mathrm{ne}}=\left(\begin{array}{ll}
\left(V_{x x}^{\mathrm{c}}-V_{x x}^{\mathrm{d}}\right) / 4 & \left(V_{x p}^{\mathrm{c}}-V_{x p}^{\mathrm{d}}\right) / 2 \\
\left(V_{x p}^{\mathrm{c}}-V_{x p}^{\mathrm{d}}\right) / 2 & V_{p p}^{\mathrm{c}}-V_{p p}^{\mathrm{d}}
\end{array}\right) .
\end{aligned}
$$

This combined covariance matrix is very similar to the covariance matrix for the amplitude and the phase quadrature of two output light beams which have been created by overlapping two continuous Gaussian light beams on a beam splitter. Note that overlapping two light beams which are differently squeezed in amplitude and phase quadrature on a beam splitter is a very common way of how continuous vari-

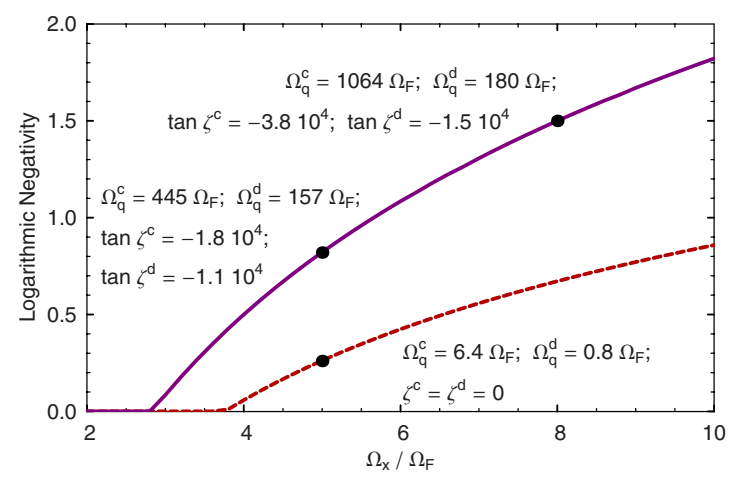

FIG. 4. (Color online) Logarithmic negativity versus $\Omega_{x} / \Omega_{F}$ maximized with respect to $\Omega_{q}^{\mathrm{c}}$ and $\Omega_{q}^{\mathrm{d}}$ using phase quadrature detection (dashed red line) as well as additionally maximized with respect to $\zeta^{\mathrm{c}}$ and $\zeta^{\mathrm{d}}$ (solid purple line). In both cases no laser noise is assumed, i.e., with $S_{a_{1} a_{1}}=S_{a_{2} a_{2}}=1$. At some positions optimal parameter values for $\Omega_{q}^{\mathrm{c}}, \Omega_{q}^{\mathrm{d}}, \zeta^{\mathrm{c}}$, and $\zeta^{\mathrm{d}}$ are given in the plot.

able entanglement is created in optics [60,61]. In the mirror case the common and the differential modes are mathematically overlapped to give the motion of each individual end mirror.

In the following we will use the logarithmic negativity, which can be found in Ref. [62] (see also references therein), as a quantitative measure of the entanglement. For our state it reads

$$
E_{\mathcal{N}}=\max \left[0,-\log _{2} 2 \sigma^{-} / \hbar\right]
$$

where we have $\sigma^{-}=\sqrt{\left(\sum-\sqrt{\Sigma^{2}-4 \operatorname{det} V_{\text {tot }}}\right) / 2}$ and $\Sigma=\operatorname{det} V_{\text {nn }}$ $+\operatorname{det} V_{\mathrm{ee}}-2 \operatorname{det} V_{\mathrm{ne}}=V_{x x}^{\mathrm{c}} V_{p p}^{\mathrm{d}}+V_{p p}^{\mathrm{c}} V_{x x}^{\mathrm{d}}-2 V_{x p}^{\mathrm{c}} V_{x p}^{\mathrm{d}}$. The higher the value of $E_{\mathcal{N}}$, the stronger the entanglement.

Recall that there exists a frequency band with sub-SQL classical noise if $\Omega_{x} / \Omega_{F}>2$. However, the existence of entanglement sets a slightly higher threshold value for this frequency ratio depending on the strength of laser noise as it is shown in Ref. [54]. We know from Sec. III B that the uncertainty product of each individual mode-common and differential-is minimal for a phase quadrature detection $\zeta^{c, d}=0$ but it has turned out that this is not the optimal choice for the preparation of entanglement. If the homodyne detection angle approaches $-\pi / 2$, each mode can become more squeezed depending on the measurement frequency.

The entanglement between the two mirrors-created by overlapping two modes-increases with the squeezing of the individual modes and with the angle separating the squeezed quadrature of the two modes. Then it is obvious that one should not observe common and differential modes via phase quadrature detection, but that there is a certain value, for each the common and the differential modes, of $\Omega_{\alpha}^{\mathrm{c}, \mathrm{d}}$ and $-\pi / 2<\zeta^{\mathrm{c}, \mathrm{d}}<0$ which is optimal for the entanglement and maximizes the logarithmic negativity (cf. the solid line in Fig. 4). These optimal parameters depend of course on the classical noise but are usually characterized by a high measurement frequency and a detection close to $-\pi / 2$ for both modes. That means that the states are totally driven by radiation pressure which is in turn monitored by reading out close to the amplitude quadrature. 
Furthermore, we have found that the laser noise-entering at the bright port and in a totally balanced interferometer only affecting the common mode-can theoretically be almost suppressed with the optimally high measurement frequency and the optimal homodyne detection angle. Therefore, the resulting maximal entanglement-represented by the solid line in Fig. 4-is independent of the laser noise. But the parameters, such as optical power and the fine tuning of the homodyne detection angle, which are required in order to reach the maximal entanglement, are different for different strengths of laser noise and are far away from any realistic situation (cf. the dots in Fig. 4).

\section{INTERFEROMETERS WITH NON-MARKOVIAN NOISE}

\section{A. Cavity with finite bandwidth}

In realistic experimental situations, the noise sources are usually not Markovian. In this section we want to generally study quantum-state preparation in the background of nonMarkovian noise sources. We start with allowing the quantum-noise spectral density to become frequency dependent.

If we consider a cavity of length $L$ with a finite cavity half-bandwidth $\gamma=\tau^{2} c /(4 L)$ and a movable high-reflective end mirror (suspended with a high quality factor and a low eigenfrequency), the quantum noise is indeed nonMarkovian and the Heisenberg equations of motion in frequency domain modify to

$$
\begin{aligned}
\hat{y}= & \sin \zeta \frac{\gamma+i \Omega}{\gamma-i \Omega} \hat{a}_{1}+\cos \zeta \times\left[\frac{\gamma+i \Omega}{\gamma-i \Omega} \hat{a}_{2}+\frac{\gamma}{\gamma-i \Omega} \frac{\alpha^{\mathrm{cav}}}{\hbar}(\hat{x}\right. \\
& \left.\left.+\hat{\xi}_{x}\right)\right] \\
\hat{x} & =-\frac{1}{m\left(\Omega^{2}+i \gamma_{m} \Omega-\omega_{m}^{2}\right)}\left[\frac{\gamma}{\gamma-i \Omega} \alpha^{\mathrm{cav}} \hat{a}_{1}+\hat{\xi}_{F}\right]
\end{aligned}
$$

where we have considered traveling waves and approximated exponential functions by rational functions in $\Omega$ by using the fact that $\gamma L / c \sim \Omega L / c \ll 1$ [9]. Note that these linearized Langevin equations in the frequency domain can also be obtained using the standard Hamiltonian as given in, e.g., Eq. (2.2) in Ref. [63]. The coupling constant is enhanced due to the storage in the cavity and therefore we have $\alpha^{\mathrm{cav}}=2 / \tau \alpha$, where $\alpha$ is given in Eq. (55) and the optical power $P$ is the one inside the cavity. The measurement output operator $\hat{y}$ includes phase quadrature fluctuations of the in-going vacuum fields, the motion of the mirror's center of mass $\hat{x}$, and classical sensing noise $\hat{\xi}_{x}$ obeying the frequency-domain correlation

$$
\left\langle\hat{\xi}_{x}(\Omega) \hat{\xi}_{x}^{\dagger}\left(\Omega^{\prime}\right)\right\rangle_{\mathrm{sym}}=2 \pi \frac{\hbar}{m \Omega_{x}^{2}} \delta\left(\Omega-\Omega^{\prime}\right) .
$$

The angle $\zeta$ denotes again the quadrature angle of the homodyne measurement performed on the output field. The motion of the test-mass's center of mass in turn is driven by radiation-pressure fluctuations as well as by classical forces $\hat{\xi}_{F}$ having the correlation

$$
\left\langle\hat{\xi}_{F}(\Omega) \hat{\xi}_{F}^{\dagger}\left(\Omega^{\prime}\right)\right\rangle_{\text {sym }}=2 \pi \hbar m \Omega_{F}^{2} \delta\left(\Omega-\Omega^{\prime}\right) .
$$

The amplitude $\hat{a}_{1}$ and the phase quadrature operator $\hat{a}_{2}$ of the in-going vacuum fields obey the correlation

$$
\left\langle\hat{a}_{i}(\Omega) \hat{a}_{j}^{\dagger}\left(\Omega^{\prime}\right)\right\rangle_{\mathrm{sym}}=\pi \delta\left(\Omega-\Omega^{\prime}\right) \delta_{i j} .
$$

Now we define the measurement frequency again as $\Omega_{q}^{\text {cav }}$ $\equiv \alpha^{\mathrm{cav}} / \sqrt{\hbar m}$, while the highest sensitivity to the displacement of the end mirror, i.e., where the position-referred quantum-noise spectral density touches the SQL, is at a different frequency, namely, at

$$
\Omega=\frac{\gamma}{\sqrt{2}} \sqrt{\sqrt{1+\left(\frac{2 \Omega^{\mathrm{cav}}}{\gamma}\right)^{2}}-1} \Omega^{\mathrm{cav}} \ll \gamma
$$

The eight zeros of the spectral density $S_{y y}$ are given by $\gamma\left( \pm a_{1} \pm i b_{1}\right)$ and $\gamma\left( \pm a_{2} \pm i b_{2}\right)$ with [for simplicity for phase quadrature detection $(\zeta=0)$ and in the free-mass limit, i.e., with $\left.\omega_{m} \rightarrow 0\right]$

$$
\begin{aligned}
& a_{1,2}=\frac{1}{2} \sqrt{\sqrt{r^{2} \mp \sqrt{2} r} \pm \frac{r}{\sqrt{2}}-1}, \\
& b_{1,2}=\frac{1}{2} \sqrt{\sqrt{r^{2} \mp \sqrt{2} r} \mp \frac{r}{\sqrt{2}}+1},
\end{aligned}
$$

where we have defined the quantity $r=\sqrt{\sqrt{\left(2 \Omega_{q}^{\text {cav }} / \gamma\right)^{4}+1}+1}$. The zeros are required for the spectral decomposition introduced in Sec. II. After straightforward algebraic manipulations, one arrives at the following conditional second-order moments (here for simplicity in the absence of classical noise):

$$
\begin{gathered}
V_{x x}=\frac{\hbar \gamma}{6 m\left(\Omega_{q}^{\text {cav }}\right)^{2}}\left(c_{1}^{3}+3 c_{1}^{2}+3 c_{1}+3 c_{3}\right), \\
V_{p p}=\frac{\hbar m \gamma^{3}}{120\left(\Omega_{q}^{\text {cav }}\right)^{2}}\left(3 c_{1}^{5}+15 c_{1}^{4}+20 c_{1}^{3}+60 c_{3}+60 c_{5}\right), \\
V_{x p}=\frac{\hbar \gamma^{2}}{16\left(\Omega_{q}^{\text {cav }}\right)^{2}} c_{1}^{2}\left(c_{1}+2\right)^{2},
\end{gathered}
$$

where the coefficients are given by

$$
c_{n}=\frac{2}{n} \operatorname{Im}\left[\left(a_{1}+i b_{1}\right)^{n}+\left(a_{2}+i b_{2}\right)^{n}-i^{n}\right] .
$$

The conditional variances given in Eqs. (80)-(82), although slightly complicated, are still analytic; we can still draw some important conclusions from these second-order moments. By expanding the quantity $U$ from Eq. (3) in terms of $\Omega_{q}^{\mathrm{cav}} / \gamma$, we obtain

$$
U=1+\frac{1}{2 \sqrt{2}} \frac{\Omega_{q}^{\mathrm{cav}}}{\gamma}+O\left(\left(\Omega_{q}^{\mathrm{cav}} / \gamma\right)^{2}\right)
$$

which reveals that, even in the quantum-noise limited case, the conditional state of the test mass cannot be pure as long 


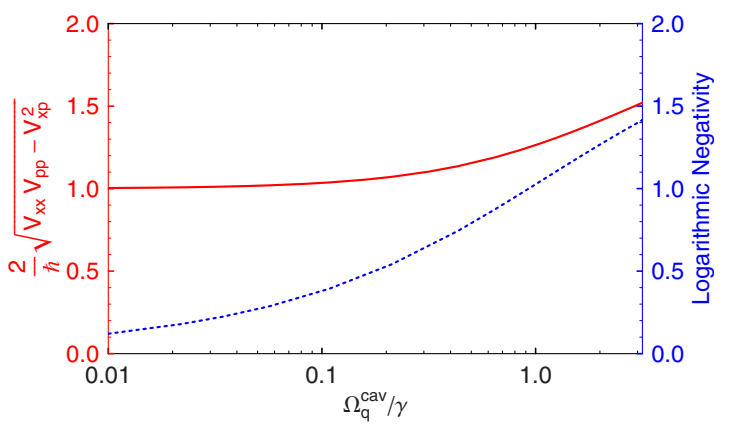

FIG. 5. (Color online) Test-mass uncertainty product $U$ (solid red line) compared to the entanglement between test mass and cavity mode (dashed blue line) both versus the dimensionless ratio $\Omega_{q}^{\text {cav }} / \gamma$ through which both quantities are totally described. No classical noise is present. Free mass limit is used, i.e., $\gamma_{m}=\omega_{m}=0$, and the phase quadrature $\zeta=0$ is detected. Entanglement is quantified by the logarithmic negativity.

as $\Omega_{q}^{\text {cav }} / \gamma>0$. This is in contrast to the Markovian limit $(\gamma$ $\rightarrow \infty)$ discussed in Sec. III B, where the conditional state is always pure in the absence of classical noise [cf. Eq. (59)]. Figure 5 shows the purity of the test mass versus the dimensionless ratio $\Omega_{q}^{\text {cav }} / \gamma$.

In the case of a finite cavity bandwidth, the light is stored inside the cavity for some time. The information carried by the light concerning the test-mass state cannot leave the cavity instantaneously and hence is not accessible for the conditioning process. Consequently, the intracavity mode needs to be taken into account for a complete characterization of the system. The residual second-order moments required for completing the corresponding $(4 \times 4)$ conditional covariance matrix can be obtained in the same way as Eqs. (80)-(82). It turns out that the composite system is indeed a pure one even though each individual system resides in a mixed state. This is a clear evidence of entanglement between the conditional states of the test mass and the cavity mode. We have also plotted the logarithmic negativity in Fig. 5. The test-mass state's mixedness and the test-mass-light entanglement increase with smaller bandwidth and with higher measurement frequency $\Omega_{q}^{\text {cav }}$. Note that the uncertainty product, as well as the logarithmic negativity, does not diverge. But Fig. 5 indicates that, as long as $\Omega_{q}^{\text {cav }} \ll \gamma$, we can neglect this effect and adiabatically eliminate the cavity mode as performed for the power-recycling cavity in Sec. IV.

Figure 6 further shows that the purity increases with higher mechanical eigenfrequency $\omega_{m}$, depending on the measurement frequency. Let us consult the following handwaving argument: with increasing $\omega_{m}$ the mechanical oscillator and the optical oscillator, which would resonate at modulation-frequency zero, become more separated in the frequency space and therefore their entanglement decreases. And with decreasing entanglement the test-mass state becomes more pure. But the regime with such high mechanical resonance frequencies is usually not available in actual $\mathrm{GW}$ detectors.

It is of course not only the entanglement between test mass and cavity mode that degenerates the purity of the mirror's quantum state. When including the two classical-noise sources from our simple model [cf. Eqs. (48) and (49)] the

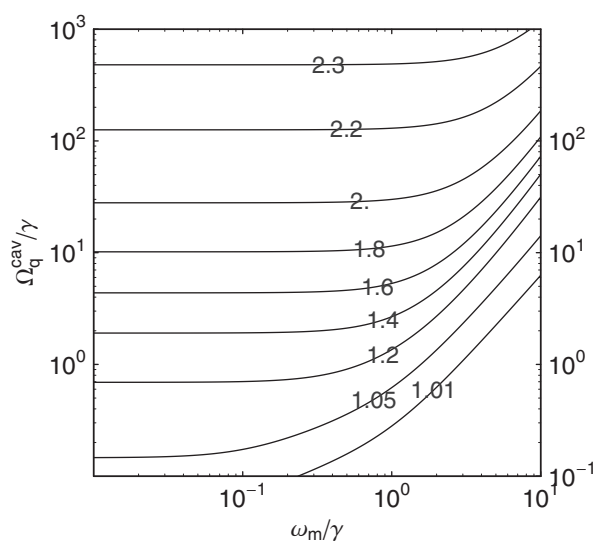

FIG. 6. Contour plot of the normalized test-mass uncertainty product given by $U=2 / \hbar \sqrt{V_{x x} V_{p p}-V_{x p}^{2}}$ versus $\Omega_{q}^{\text {cav }} / \gamma$ and $\omega_{m} / \gamma$ for quantum noise only. Again phase quadrature $(\zeta=0)$ is detected.

conditional state becomes more and more mixed with an increasing classical-noise level as shown in Fig. 7 which is not due to the entanglement- the classical noise rather destroys the quantum entanglement between the test mass and the cavity mode. The purity also depends on the ratio between the measurement frequency and the optical bandwidth. Recall that the classical force noise increases with higher $\Omega_{F}$ while the classical sensing noise increases with lower $\Omega_{x}$. If we only take sensing noise into account we know for sure that the motion of the test mass is solely driven by quantum back-action noise. A high sensing noise level randomizes the measurement record and hence the delimitated accessibility
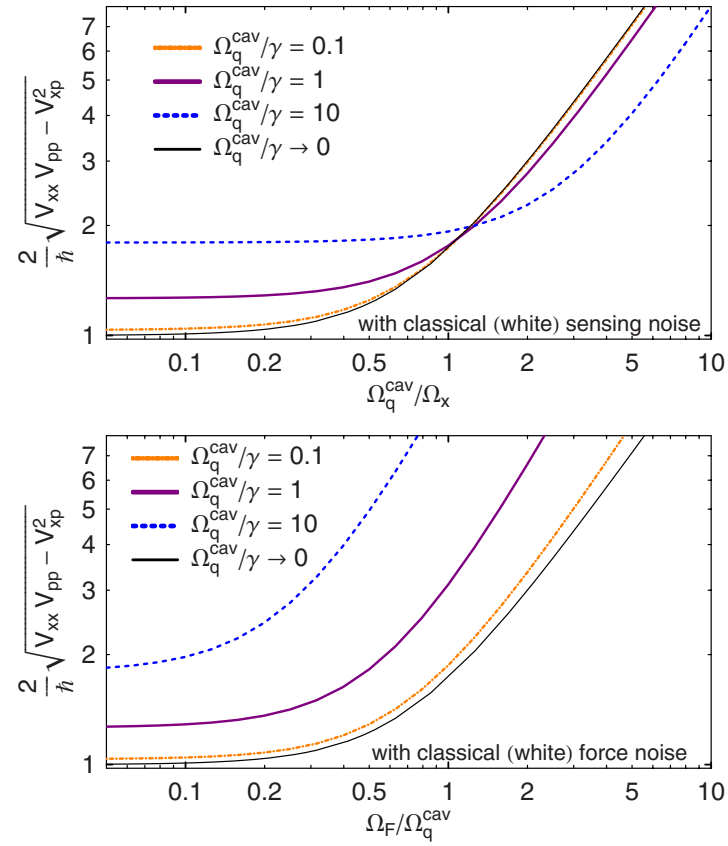

FIG. 7. (Color online) Test-mass uncertainty product $U$ versus classical sensing noise and without force noise (upper panel) as well as versus classical force noise without classical sensing noise (lower panel) both for different examples of ratios between measurement frequency and bandwidth. Free mass limit is used, i.e., $\omega_{m}=0$, as well as phase quadrature detection $\zeta=0$. 
of the intracavity field due to the finite bandwidth is insignificant. Consequently all curves roughly coincide for $\Omega_{q}^{\text {cav }}$ $\gtrsim \Omega_{x}$ in the upper panel of Fig. 7 .

Note that in the planned Advanced LIGO detector [5], the measurement frequency $\Omega_{q}^{\mathrm{cav}}$ is planned to roughly coincide with the cavity half-bandwidth at $\gamma /(2 \pi) \sim 100 \mathrm{~Hz}$-corresponding to the solid lines in Fig. 7. Furthermore, we expect the suspension thermal noise to have a $\Omega_{F} /(2 \pi) \sim 30-40 \mathrm{~Hz}$-that would be less than $\Omega_{q}^{\text {cav }} / 2$-but the coating thermal noise may provide a $\Omega_{x}$ that only coincides with $\Omega_{F}$ or is just marginally higher. We can infer form Fig. 7 that the quantum state of the interferometer's differential mode is mainly constrained by classical sensing noise which entails a lower bound of $U \geqslant 5$ and gives an effective occupation number of $\mathcal{N}_{\text {eff }} \geq 2$. For a more detailed discussion see Sec. VI.

\section{B. Detuned cavity}

A cavity which is detuned by $\Delta$ from the carrier's frequency makes the power inside the cavity also dependent on the motion of the test-mass mirrors. This creates an optical spring [64] or an optical antispring both shifting the (free) mechanical and the (free) optical resonance frequencies in the complex plane. In future GW detectors such as Advanced LIGO [5], the optical spring effect will be used to up shift the real part of the mechanical resonance frequency into the detection band. Recall that the optical spring, as well as the optical antispring, usually introduces instability to the system which has to be cured with an appropriate linear feedback control [51]. But it is straightforward to show that the conditional covariance matrix does not change under any ideal linear feedback control —as long as the closed-loop system is stable.

The Heisenberg equations of motion for such a system can be found in many previous works (see, e.g., Eqs. (39)(41) in Ref. [65]). Unfortunately, analytic expressions for the conditional covariance matrix are cumbersome, and we only report numerical results. We also restrict ourselves to quantum noise and reading out the phase quadrature, i.e., $\zeta=0$ (cf. Eq. (3.2) in Ref. [51]). The measurement frequency $\Omega_{q}^{\text {cav }}$ is defined in Eq. (77).

Figure 8 shows that if detuning the cavity from the carrier frequency properly, the purity can be increased which comes from the fact that the quantum entanglement between test mass and cavity mode is decreased. In the regime of a blue detuned cavity $(\Delta>0)$ - producing an optical spring —and for $\Omega_{q}^{\text {cav }}<\gamma$, Fig. 8 simply agrees with Fig. 6 . Here at fixed measurement frequency $\Omega_{q}^{\text {cav }}<\gamma$ a higher detuning $\Delta$ gives a less shifted mechanical resonance, so-called optomechanical resonance, and at the same time it corresponds to a higher optical resonance. Therefore, again the two oscillators are more separated in the frequency space and their entanglement decreases. Interestingly, for higher $\Omega_{q}^{\text {cav }}$ the test-mass state could locally appear more pure in the red detuned cavity regime, i.e., at a certain $\Delta<0$, which produces an optical antispring. Note further that the uncertainty product diverges for an infinitely red detuned cavity $(\Delta \rightarrow-\infty)$. For these facts we unfortunately have not found any intuitive explanation.

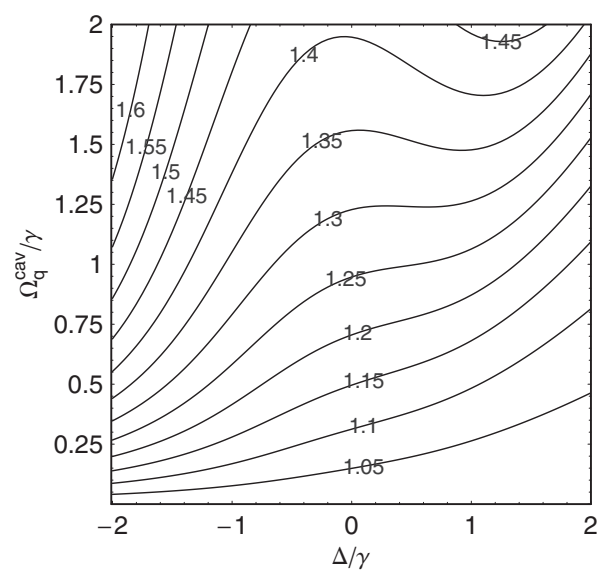

FIG. 8. Contour plot of the normalized test-mass uncertainty product given by $U=2 / \hbar \sqrt{V_{x x} V_{p p}-V_{x p}^{2}}$ versus the ratio between detuning $\Delta$ and bandwidth and $\Omega_{q}^{\text {cav }} / \gamma$ for quantum noise only. Free mass limit is used, i.e., $\omega_{m}=0$, as well as phase quadrature detection $\zeta=0$.

This will be a subject of further investigation.

\section{Non-Markovian classical noise}

In the following we consider a more realistic example configuration involving multiple colored classical-noise sources. The classical-noise contributions are highly nonMarkovian and they tend to rise fast in the low-frequency regime, which is ignored by a simple Markovian noise model. First we restrict ourselves to an idealized noise budget of an advanced interferometric GW detector, shown in the lower panel of Fig. 9. Here only the dominating force and sensing noise sources are considered. Additionally we assume that the gravity gradient noise can be suppressed completely through monitoring the ground's motion. Especially seismic noise dominates the entire spectrum below $10 \mathrm{~Hz}$. In order to apply the numerical Wiener filter procedure (cf. Sec. II), all classical-noise spectra need to be approximated by rational functions of $\Omega^{2}$. This is illustrated by Fig. 9 (upper panel) where the seismic noise spectral density, preestimated by the simulation tool Bench [66], is approximated accordingly. The seismic noise spectrum is constant below $0.25 \mathrm{~Hz}$, then it drops as $\sim 1 / f^{6}$ between 0.25 and $2 \mathrm{~Hz}$, and finally it drops as $\sim 1 / f^{10}$ above $2 \mathrm{~Hz}$. The suspension thermal noise constitutes a second force noise contribution which drops as $\sim 1 / f^{5 / 2}$ above the pendulum eigenfrequency (at $1 \mathrm{~Hz}$ ) and it intersects the SQL at $20 \mathrm{~Hz}$. Such a frequency dependence presumes structural damping. Above 3 $\mathrm{Hz}$ the internal thermal noise follows $\sim 1 / f^{1 / 2}$ and it intersects the SQL at $500 \mathrm{~Hz}$. We have employed the Padé expansion in order to simulate the behavior of the spectral densities (cf. lower panel of Fig. 9).

It should be emphasized that the conditional second-order moments can diverge if the sensing noise rises toward low frequencies, and therefore a cutoff frequency must be chosen carefully. This issue is illustrated by Fig. 10 where the cutoff frequency of the sensing noise is varied, while the classical force noise contributions are held fixed. This divergence can 

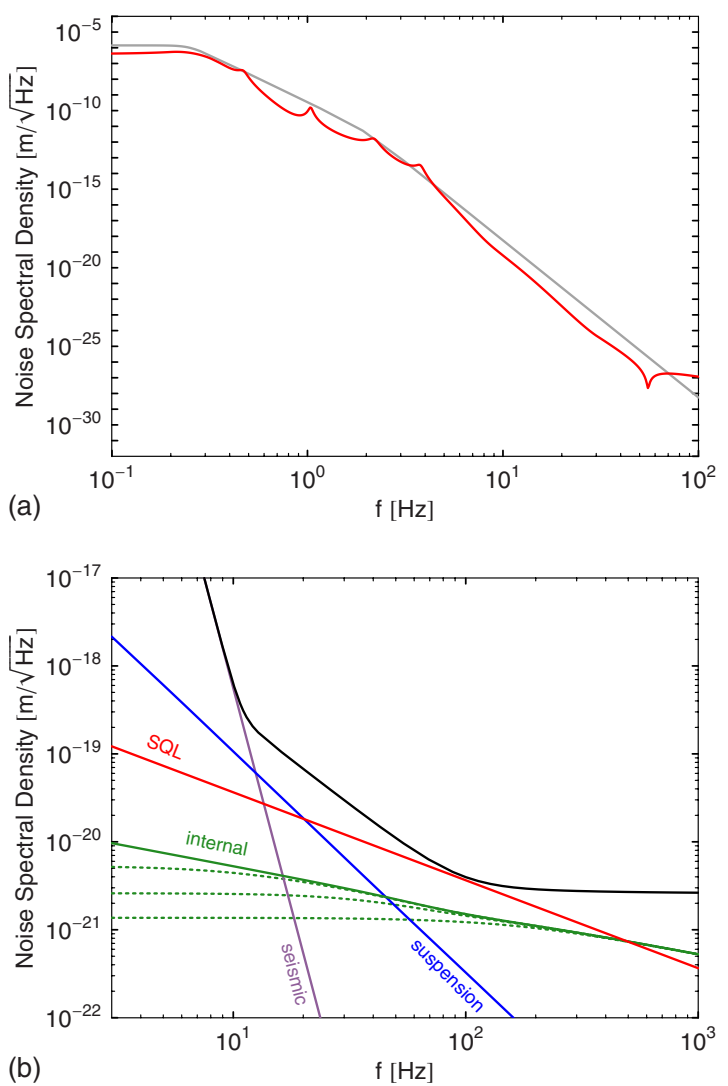

FIG. 9. (Color online) Upper panel: seismic noise pre-estimated by the simulation tool Bench [66] (red curve) and a fit by rational function (gray smooth line). Lower panel: straw man classical-noise budget of an advanced interferometric GW detector. Seismic (violet, follows $\sim 1 / f^{1} 0$ ), suspension thermal (blue, follows $\sim 1 / f^{5 / 2}$ ), and internal thermal (green, follows $\sim 1 / f^{1 / 2}$, dashed for different cutoff frequencies) noise spectra are shown as well as the total noise (black)_including Markovian quantum noise with phase quadrature readout. For the thermal noise sources we employed a Padé approximation that generated the $1 / f$ spectra between 0.1 and $2 \mathrm{~Hz}$.

be explained as follows: for a free mass, the effect of radiation-pressure noise diverges toward low frequencies. Hence the boundedness of the conditional variances depends

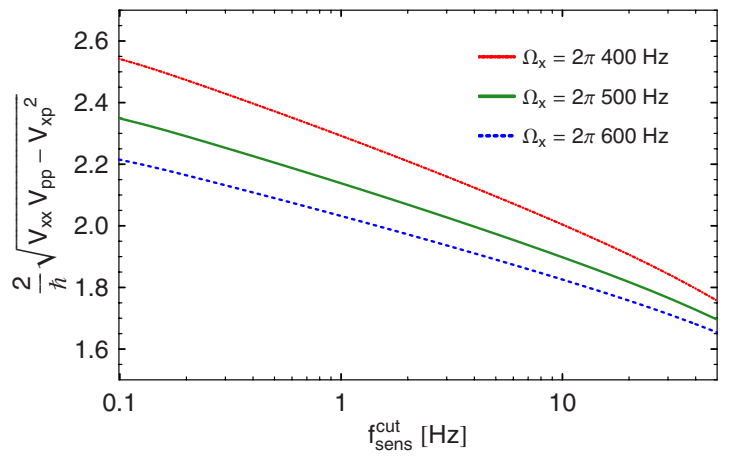

FIG. 10. (Color online) Uncertainty product of the conditional state versus sensing noise cutoff frequency for three different sensing noise levels. Force noise contributions are the same as in Fig. 9. The second-order moments formally diverge when downshifting the cutoff frequency. crucially on the motion of the test mass at low frequencies. Furthermore the mirror thermal noise with $S(f) \sim 1 / f$ formally leads to a logarithmic divergence. In the case of a real experimental setup the mirrors are suspended as pendulums and the mirror thermal noise should also exhibit a lowfrequency cutoff-but more importantly the low-frequency noise will be canceled out in a subsequent verification stage as it will be shown in a forthcoming paper [55]. Such a cancellation arises from the fact that for the mirror thermal noise with frequencies lower than the inverse of the sum of the preparation-stage and verification-stage measurement time scales, their contributions to errors in the preparation and verification measurements are the same and therefore cancel out when the two sets of data are compared with each other. This argument justifies an increase in the cutoff frequency to a level of around $3 \mathrm{~Hz}$.

\section{ADVANCED LIGO CONFIGURATIONS}

In this section we will investigate the performance of the planned Advanced LIGO detector [5], a second generation GW observatory, toward the preparation of test-mass quantum states. It is planned that this large-scale laser interferometer (cf. Fig. 1) - 4-km-long arm cavities consisting of $40 \mathrm{~kg}$ mirrors-starts its operation in 2014. It will be nearly quantum noise limited in most of its frequency band $(10 \mathrm{~Hz}-10$ $\mathrm{kHz}$ ) and will operate near or at its SQL. Our previous investigations in Secs. III and V have suggested that such a SQL sensitivity allows to prepare nearly Heisenberg-limited quantum states of macroscopic test masses. Note that we will consider the differential mode of the interferometer's four movable arm-cavity mirrors which is equivalent to a single movable mirror in a single detuned cavity [65]—with one quarter of the mass of each individual mirror, i.e., $10 \mathrm{~kg}$.

The classical-noise budget of the Advanced LIGO detector has been estimated by the simulation tool Bench [66].

We choose the same type of spectra as for the example configuration in Sec. V C, i.e., with identical power laws and cutoffs in frequency, and adjust the parameters such that the predicted Advanced LIGO classical-noise budget is well approximated. In contrast to Sec. V C, Advanced LIGO comprises finite-bandwidth cavities, which gives rise to a nonMarkovian quantum noise. Moreover, the detuned signalrecycling technique introduces even the optical spring into the dynamics of the mirrors just as in the case of a detuned cavity (cf. Sec. V A). As an example, the quantum noise of the Advanced LIGO broadband configuration, which is optimal for the detection of neuron star binary inspirals, is plotted in the upper panel of Fig. 11.

We have carried out a full parameter search over the space of signal-recycling parameters in order to optimize the configuration with respect to the uncertainty product of the conditional state. We have fixed the arm-cavity half-bandwidth $\gamma \approx 2 \pi \times 100 \mathrm{~Hz}$ and the characteristic frequency of the system (as defined in Eq. (20) in Ref. [65] and given by $\left.\left(\iota_{c}\right)^{1 / 3}=\left[\left(\Omega_{q}^{\text {cav }}\right)^{2} \gamma\right]^{1 / 3} \approx 2 \pi \times 100 \mathrm{~Hz}\right)$, which is basically determined by the fixed circulating optical power of $800 \mathrm{~kW}$ in the arm cavities. Furthermore, we have only considered a homodyne detection at the Advanced LIGO broadband con- 

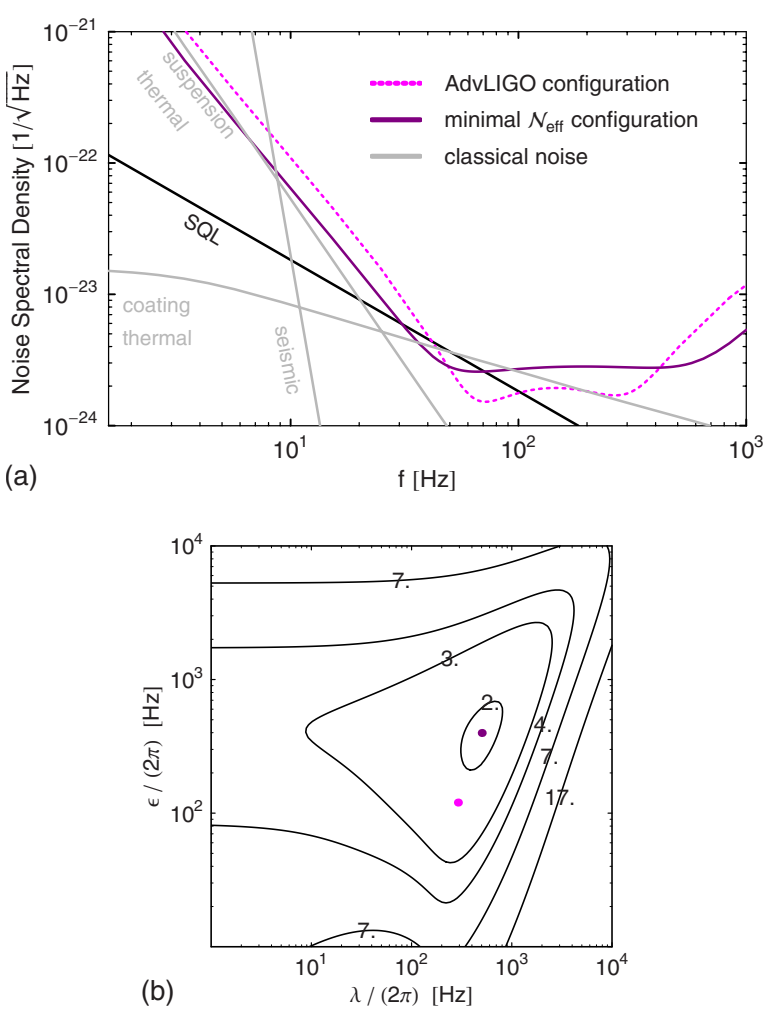

FIG. 11. (Color online) Upper panel: spectral densities of main noise sources present in the Advanced LIGO detector: seismic, suspension thermal, and internal thermal noise (as marked), as well as two examples of the quantum noise (magenta solid and purple dashed lines). Pre-estimated classical non-Markovian noise budget is fitted by rational functions with characteristic spectra as in Fig. 9. Lower panel: effective occupation number $\mathcal{N}_{\text {eff }}$ of conditional state (of the differential mirror mode) versus effective detuning $\lambda$ and effective bandwidth $\epsilon$. The dots mark the Advanced LIGO broadband configuration state (magenta) and lowest occupation number state (purple, within the two contours) when $\zeta=0.7 \pi$.

figuration quadrature, i.e., $\zeta=0.7 \pi$. The result of this optimization is shown in the lower panel of Fig. 11 which depicts the effective occupation number $\mathcal{N}_{\text {eff }}$ as introduced in Appendix A-versus the effective detuning $\lambda$ and the effective bandwidth $\epsilon$-these two quantities are defined in Eq. (18) in Ref. [65]. It clearly shows that the purity of the conditional state benefits from a restoring optical spring, i.e., a positive detuning facilitates the preparation of macroscopic quantum states as it was shown before. Note that increasing the effective bandwidth $\epsilon$ to some extent gives rise to an additional improvement, which has also been clarified before. The Advanced LIGO broadband configuration with $\lambda=2 \pi$ $\times 290 \mathrm{~Hz}$ and $\epsilon=2 \pi \times 120 \mathrm{~Hz}$ is marked with a dot in the lower panel of Fig. 11 and gives $\mathcal{N}_{\text {eff }} \approx 2.2$, while the other dot marks the purest state at $\lambda=2 \pi \times 500 \mathrm{~Hz}$ and $\epsilon=2 \pi$ $\times 400 \mathrm{~Hz}$ which gives $\mathcal{N}_{\mathrm{eff}} \approx 1.9$. An additional optimization of the homodyne detection angle decreases this number only marginal.

Aside from the currently estimated classical-noise budget, a more optimistic scenario [67] has been investigated, in which the seismic and suspension thermal noises are reduced by a factor of 10 , while the coating thermal noise is lowered
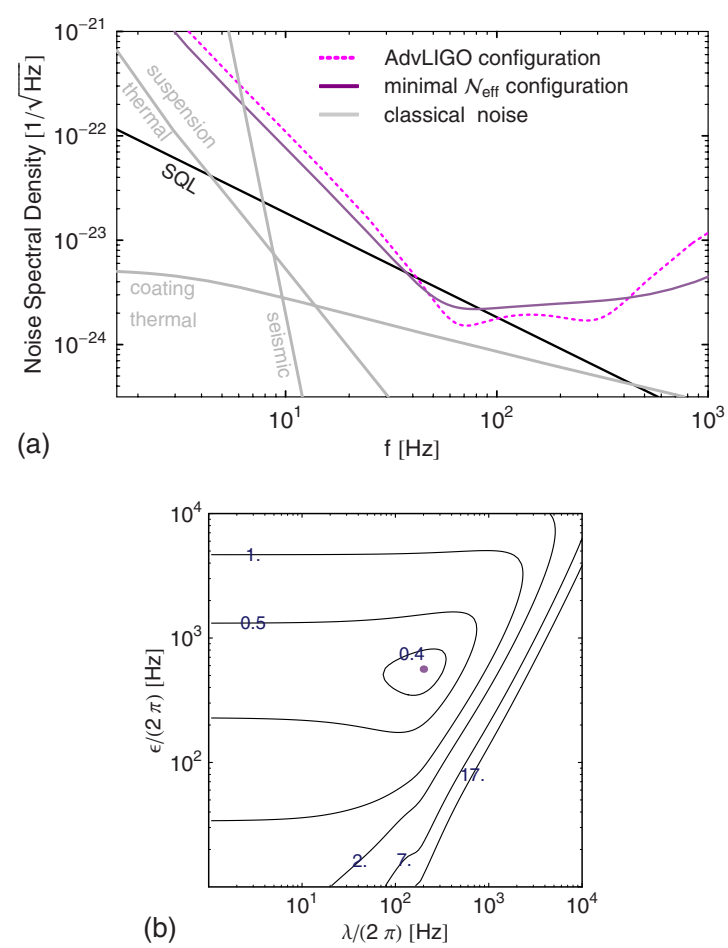

FIG. 12. (Color online) Upper panel: spectral densities of main noise sources present in an improved Advanced LIGO detector. Lower panel: effective occupation number $\mathcal{N}_{\text {eff }}$ of the differential mirror mode's conditional state versus effective detuning $\lambda$ and effective bandwidth $\epsilon$ for phase quadrature detection. The dot marks the lowest occupation number state.

by a factor of 3 (in amplitude). Here the cutoff frequencies remain the same. A rough optimization has revealed that the minimal achievable effective occupation number drops down to $\mathcal{N}_{\text {eff }} \approx 0.38$ (cf. Fig. 12) for phase quadrature detection. Further major improvements regarding the classical-noise level in the Advanced LIGO detector-especially the laser noise-will even allow to entangle the cavity mirrors in the north with those in the east arm.

\section{CONCLUSION}

This paper has been devoted in great detail to a survey of the first principles in the preparation of macroscopic Gaussian quantum state of nonmassless objects. We have motivated and introduced the Wiener filter method in this context - as an advantage over the SMEs — and have given a simple analytical expression for the covariance matrix of a system under any continuous linear Markovian measurement process. We have shown that in absence of any additional noise, the conditional state is totally determined by the measurement noise. Moreover, the purity of the conditional state is even equal to the purity of the underlying measurement process [cf. Eq. (45)]. This provides an important insight into the understanding of conditional states which was probably not communicated before.

In Markovian measurements with noncorrelated shot and radiation-pressure noise, we have shown that the effective occupation number of the conditional state is connected to 
the factor at which the device beats the SQL, i.e., to the bandwidth within which the classical noise is below the SQL [cf. Eqs. (51) and (60)]. For $S_{\mathrm{cl}}<S_{\mathrm{SQL}}$ around the frequency $\Omega_{\mathrm{cl}}$, at which the two classical-noise spectra intersect, we find $\mathcal{N}_{\text {eff }}<1 / 2$. We have shown that neither a balanced homodyne detection of a nonphase quadrature nor input squeezing would help to get a more pure state-but they can significantly steer the shape of the conditional state, e.g., the test-mass squeezing.

Furthermore, we have motivated that a simple powerrecycled Michelson interferometer is the ideal device to prepare macroscopic entanglement [54]. We have shown that the existence of entanglement in position and momentum between the two end mirrors is closely related to the factor at which the classical noise beats the SQL: a quantum measurement with a flexible but frequency-independent homodyne detection angle and no restriction to the optical power as an example theoretically requires the classical noise to be at least a factor of 1.5 below the free-mass SQL at a certain sideband frequency.

Moreover, we studied mirror quantum-state preparation in non-Markovian quantum-measurement systems. In the first instance we have considered the conditional quantum state of a test mass inside a finite-bandwidth system. It has been demonstrated that even a quantum-noise limited configuration does not allow the preparation of a minimumHeisenberg uncertainty state, due to quantum entanglement between the test mass and the cavity mode, which has a nonzero lifetime.

It has been pointed out that the purity of a conditional quantum state of macroscopic test masses can benefit from introducing an optical spring. This has been verified numerically for the quantum-noise limited regime.

Finally, we have optimized the effective occupation number of the differential mode of the planned Advanced LIGO GW detector in the presence of pre-estimated realistic decoherence processes. It has been confirmed that already a moderately reduced classical-noise budget, such as for an improved Advanced LIGO detector, allows us to prepare a nearly pure quantum state of the mechanical mode under consideration. More concrete, we have shown that an occupation number of $\approx 2.2$ is readily reachable by the baseline design, a moderate shift in optical parameters can achieve $\approx 1.9$, while a moderate enhancement in classical-noise budget could achieve $\approx 0.38$. Third-generation GW detectors or prototype interferometers specifically designed for testing macroscopic quantum mechanics would be able to surpass this moderate enhancement of Advanced LIGO and reach deep into the quantum regime.

\section{ACKNOWLEDGMENTS}

We thank all the members of the AEI-Caltech-MIT MQM discussion group for very useful discussions. We thank K. S. Thorne for initiating this research project and V. B. Braginsky for important critical comments. Research of H.M.-E., K.S., and Y.C. is supported by the Alexander von Humboldt Foundation's Sofja Kovalevskaja Programme. Y.C. and K.S. are also supported by the National Science Foundation
(NSF) Grant No. PHY-0653653 and No. PHY-0601459, as well as the David and Barbara Groce startup fund at Caltech. Research of H.R. and R.S. is supported by the Deutsche Forschungsgemeinschaft through SFB No. 407. K.S. is also supported by the Japan Society for the Promotion of Science (JSPS). Y.M. is supported by NSF Grant No. PHY-0601459 and No. PHY-0653653, NASA Grant No. NNX07AH06G and No. NNG04GK98G, and the Brinson Foundation. Research of C.L. is supported by National Science Foundation Grant No. PHY-0099568 and No. PHY-0601459.

\section{APPENDIX A: EFFECTIVE OCCUPATION NUMBER}

The normalized uncertainty product of a Gaussian state [cf. Eq. (3)] corresponds to the area of the state in phase space and is a true measure of the purity and therefore a reasonable measure of the quantumness of the state. Even though it is probably more common to used the linear entropy,

$$
\mathcal{S}_{\text {lin }}=1-\operatorname{tr} \hat{\rho}^{2}=1-1 / U
$$

as a measure of the mixedness of a given state. But since these quantities are simply connected one can always choose one of them to characterize the state.

Trying to reconstruct, as commonly done, the number of quanta, the so-called occupation number, may not always be the most fundamental figure of merit: squeezed states, for example, can have high occupation numbers, yet they should be considered probably more quantum than vacuum states. Moreover, the definition of an occupation number requires a well-defined real-valued eigenfrequency, which does not always naturally exist. Fortunately, the uncertainty product can be converted back into an effective occupation number by using the relation

$$
\mathcal{N}_{\text {eff }}=U / 2-1 / 2 \text {. }
$$

If a state has no correlation in position and momentum, i.e., $V_{x p}=0$, this effective occupation number should be interpreted as follows: suppose that the variances in position and momentum are given and produced by a perfect harmonic oscillator in a quadratic potential having an arbitrary but real-valued eigenfrequency $\omega$. Then the effective occupation number is obtained by minimizing the total energy divided by the energy of each quanta with respect to that eigenfrequency $\omega$. This strategy reads

$$
\mathcal{N}_{\text {eff }}=\min _{\omega}\left\{\frac{1}{\hbar \omega}\left(\frac{V_{p p}}{2 m}+\frac{m \omega^{2} V_{x x}}{2}\right)-\frac{1}{2}\right\},
$$

where the minimum is achieved at the effective eigenfrequency

$$
\omega=\omega_{\mathrm{eff}} \equiv \sqrt{\frac{V_{p p}}{m^{2} V_{x x}}} .
$$

Thus, the effective occupation number is the minimal occupation number one could obtain when assuming to have a harmonic oscillator with no correlation in position and momentum and an effective eigenfrequency as given in Eq (A4). 
For a state with correlation in position and momentum, the effective occupation number still gives the minimal occupation but with respect to two other orthogonal quadratures which are not position and momentum. Moreover, the effective occupation number is an interesting quantity because it in fact determines the von Neumann entropy of a state [68] as given by

$$
\mathcal{S}_{\mathrm{vN}}=-\operatorname{tr}[\hat{\rho} \ln \rho]=\left(\mathcal{N}_{\text {eff }}+1\right) \ln \left(\mathcal{N}_{\text {eff }}+1\right)-\mathcal{N}_{\text {eff }} \ln \mathcal{N}_{\text {eff }}
$$

In principle one can freely choose between $U, \mathcal{S}_{\text {lin }}, \mathcal{N}_{\text {eff }}$, and $\mathcal{S}_{\mathrm{vN}}$ to describe the purity of a Gaussian state.

\section{APPENDIX B: QUANTUM WIENER FILTER}

Here we directly evaluate the conditional generating functional involving the linear observables $\hat{x}_{l}(t)$,

$$
\begin{aligned}
J & \equiv \operatorname{tr}\left[\hat{\rho}^{\text {cond }} \exp \left(i \sum_{l} \alpha_{l} \hat{x}_{l}(t)\right)\right] \\
& =\operatorname{tr}\left[\hat{\rho} \mathcal{P}_{\left[\hat{y}\left(t^{\prime}\right)=y(t) \mid t^{\prime}<t\right]} \exp \left(i \sum_{l} \alpha_{l} \hat{x}_{l}(t)\right)\right],
\end{aligned}
$$

with

$$
\begin{gathered}
-\left.i \frac{\partial}{\partial \alpha_{l}}\right|_{\forall i \alpha_{i}=0} J=x_{l}^{\mathrm{cond}}(t), \\
\left.(-i)^{2} \frac{\partial^{2}}{\partial \alpha_{l} \partial \alpha_{m}}\right|_{\forall i \alpha_{i}=0} J=V_{x_{l} x_{m}}^{\text {cond }}+x_{l}^{\mathrm{cond}} x_{m}^{\text {cond }} .
\end{gathered}
$$

If we then write the projection operator as a path integral,

$$
\mathcal{P}_{\left[\hat{y}\left(t^{\prime}\right)=y(t) \mid t^{\prime}<t\right]} \propto \int D[k] \exp \left(i \int_{-\infty}^{t} d t^{\prime} k\left(t^{\prime}\right)\left[\hat{y}\left(t^{\prime}\right)-y\left(t^{\prime}\right)\right]\right),
$$

we have

$$
\begin{aligned}
J \propto & \int D[k] \operatorname{tr}\left[\hat { \rho } \operatorname { e x p } \left(i \sum_{l} \alpha_{l} \hat{x}_{l}(t)+i \int_{-\infty}^{t} d t^{\prime} k\left(t^{\prime}\right)\left[\hat{y}\left(t^{\prime}\right)\right.\right.\right. \\
& \left.\left.\left.-y\left(t^{\prime}\right)\right]\right)\right] .
\end{aligned}
$$

For a Gaussian state $\hat{\rho}$ and any linear observable $\hat{x}=\hat{x}^{\dagger}$ if $\operatorname{tr}[\hat{\rho} \hat{x}]=0$, then we always have

$$
\operatorname{tr}\left[\hat{\rho} e^{i \hat{x}}\right]=e^{-\left\langle\hat{x}^{2}\right\rangle / 2}
$$

Using this property, we obtain

$$
\begin{aligned}
J \propto & \int D[k] \exp \left\{-\left\langle\left[\sum_{l} \alpha_{l} \hat{x}_{l}(t)\right.\right.\right. \\
& \left.\left.\left.+\int_{-\infty}^{t} d t^{\prime} k\left(t^{\prime}\right) \hat{y}\left(t^{\prime}\right)\right]^{2}\right\rangle / 2\right\} \exp \left[-i \int_{-\infty}^{t} d t^{\prime} k\left(t^{\prime}\right) y\left(t^{\prime}\right)\right] .
\end{aligned}
$$

Suppose Eqs. (12) and (13) hold, i.e.,

$$
\begin{aligned}
\hat{x}_{l}(t) & =\hat{R}_{l}(t)+\int_{-\infty}^{t} d t^{\prime} K_{l}\left(t-t^{\prime}\right) \hat{y}\left(t^{\prime}\right), \quad\left\langle\hat{R}_{l}(t) \hat{y}\left(t^{\prime}\right)\right\rangle \\
& =0, \quad \forall t^{\prime}<t,
\end{aligned}
$$

then

$$
\begin{gathered}
\left\langle\left[\sum_{l} \alpha_{l} \hat{x}_{l}(t)+\int_{-\infty}^{t} d t^{\prime} k\left(t^{\prime}\right) \hat{y}\left(t^{\prime}\right)\right]^{2}\right\rangle=\sum_{l m} \alpha_{l} \alpha_{m}\left\langle\hat{R}_{l}(t) \hat{R}_{m}(t)\right\rangle \\
\quad+\int_{-\infty}^{t} \int_{-\infty}^{t} d t^{\prime} d t^{\prime \prime} \tilde{k}\left(t^{\prime}\right) \tilde{k}\left(t^{\prime \prime}\right)\left\langle\hat{y}\left(t^{\prime}\right) \hat{y}\left(t^{\prime \prime}\right)\right\rangle,
\end{gathered}
$$

where we have defined

$$
\tilde{k}\left(t^{\prime}\right)=k\left(t^{\prime}\right)+\sum_{l} \alpha_{l} K_{l}\left(t-t^{\prime}\right)
$$

Using $\tilde{k}(t)$ as the new integration variable, $J$ can be rewritten as

$$
\begin{aligned}
& J \propto \exp \left[-\frac{1}{2} \sum_{l m} \alpha_{l} \alpha_{m}\left\langle\hat{R}_{l}(t) \hat{R}_{m}(t)\right\rangle\right] \exp \left[i \int_{-\infty}^{t} d t^{\prime} \sum_{l} \alpha_{l} K_{l}(t\right. \\
& \left.\left.-t^{\prime}\right) y\left(t^{\prime}\right)\right]
\end{aligned}
$$

which justifies Eqs. (14) and (15).
[1] B. Abbott et al., Nucl. Instrum. Methods Phys. Res. A 517, 154 (2004).

[2] L. D. Fiore and The VIRGO Collaboration, Class. Quantum Grav. 19, 1421 (2002).

[3] B. Willke et al., Class. Quantum Grav. 19, 1377 (2002).

[4] M. Ando and The TAMA Collaboration, Phys. Rev. Lett. 86, 3950 (2001).

[5] Advanced LIGO Reference Design, LIGO Technical Report
No. M060056, 2007, (unpublished) http:// www.ligo.caltech.edu/docs/M/M060056-08/M060056-08.pdf

[6] C. M. Caves, K. S. Thorne, R. W. Drever, V. D. Sandberg, and M. Zimmermann, Rev. Mod. Phys. 52, 341 (1980).

[7] V. B. Braginsky and F. Y. Khalili, Quantum Measurement (Cambridge University Press, Cambridge, 1999).

[8] V. B. Braginsky, Sov. Phys. JETP 26, 831 (1968).

[9] H. J. Kimble, Y. Levin, A. B. Matsko, K. S. Thorne, and S. P. 
Vyatchanin, Phys. Rev. D 65, 022002 (2001).

[10] J.-M. Courty, A. Heidmann, and M. Pinard, Phys. Rev. Lett. 90, 083601 (2003).

[11] F. Y. Khalili, Phys. Lett. A 298, 308 (2002).

[12] P. Purdue, Phys. Rev. D 66, 022001 (2002).

[13] P. Purdue and Y. Chen, Phys. Rev. D 66, 122004 (2002).

[14] Y. Chen, Phys. Rev. D 67, 122004 (2003).

[15] J. Harms, Y. Chen, S. Chelkowski, A. Franzen, H. Vahlbruch, K. Danzmann, and R. Schnabel, Phys. Rev. D 68, 042001 (2003).

[16] F. Y. Khalili, Phys. Lett. A 317, 169 (2003).

[17] S. L. Danilishin, Phys. Rev. D 69, 102003 (2004).

[18] A. Buonanno and Y. Chen, Phys. Rev. D 69, 102004 (2004).

[19] T. Corbitt, N. Mavalvala, and S. E. Whitcomb, Phys. Rev. D 70, 022002 (2004).

[20] S. L. Danilishin and F. Y. Khalili, Phys. Rev. D 73, 022002 (2006).

[21] F. Y. Khalili, Phys. Rev. D 76, 102002 (2007).

[22] F. Y. Khalili, Phys. Rev. D 77, 062003 (2008).

[23] H. Rehbein, H. Müller-Ebhardt, K. Somiya, C. Li, R. Schnabel, K. Danzmann, and Y. Chen, Phys. Rev. D 76, 062002 (2007).

[24] H. Rehbein, H. Müller-Ebhardt, K. Somiya, S. L. Danilishin, R. Schnabel, K. Danzmann, and Y. Chen, Phys. Rev. D 78, 062003 (2008).

[25] S. Miyoki, T. Uchiyama, K. Yamamoto, H. Hayakawa, K. Kasahara, H. Ishitsuka, M. Ohashi, K. Kuroda, and D. Tatsumi, Class. Quantum Grav. 21, 1173 (2004).

[26] A. Bunkowski, O. Burmeister, D. Friedrich, K. Danzmann, and R. Schnabel, Class. Quantum Grav. 23, 7297 (2006).

[27] R. O'Shaughnessy, Class. Quantum Grav. 23, 7627 (2006).

[28] M. Bondarescu and K. S. Thorne, Phys. Rev. D 74, 082003 (2006).

[29] M. Evans, S. Ballmer, M. Fejer, P. Fritschel, G. Harry, and G. Ogin, Phys. Rev. D 78, 102003 (2008).

[30] H. J. Kimble, B. L. Lev, and J. Ye, Phys. Rev. Lett. 101, 260602 (2008).

[31] B. Abbott et al., New J. Phys. 11, 073032 (2009).

[32] P. F. Cohadon, A. Heidmann, and M. Pinard, Phys. Rev. Lett. 83, 3174 (1999).

[33] J.-M. Courty, A. Heidmann, and M. Pinard, Eur. Phys. J. D 17, 399 (2001).

[34] A. Naik, O. Buu, M. D. LaHaye, A. D. Armour, A. A. Clerk, M. P. Blencowe, and K. C. Schwab, Nature (London) 443, 193 (2006).

[35] D. Kleckner and D. Bouwmeester, Nature (London) 444, 75 (2006).

[36] T. Corbitt, C. Wipf, T. Bodiya, D. Ottaway, D. Sigg, N. Smith, S. Whitcomb, and N. Mavalvala, Phys. Rev. Lett. 99, 160801 (2007).

[37] M. Poggio, C. L. Degen, H. J. Mamin, and D. Rugar, Phys. Rev. Lett. 99, 017201 (2007).

[38] C. M. Mow-Lowry, A. J. Mullavey, S. Gossler, M. B. Gray, and D. E. McClelland, Phys. Rev. Lett. 100, 010801 (2008).

[39] A. Vinante et al., Phys. Rev. Lett. 101, 033601 (2008).

[40] S. Mancini, D. Vitali, and P. Tombesi, Phys. Rev. Lett. 80, 688 (1998).

[41] S. Danilishin, H. Müller-Ebhardt, H. Rehbein, K. Somiya, R. Schnabel, K. Danzmann, T. Corbitt, C. Wipf, N. Mavalvala, and Y. Chen, e-print arXiv:0809.2024.

[42] C. H. Metzger and K. Karrai, Nature (London) 432, 1002 (2004).

[43] S. Gigan, H. Böhm, M. Paternostro, F. Blaser, G. Langer, J. Hertzberg, K. Schwab, D. Bäuerle, M. Aspelmeyer, and A. Zeilinger, Nature (London) 444, 67 (2006).

[44] O. Arcizet, P.-F. Cohadon, T. Briant, M. Pinard, and A. Heidmann, Nature (London) 444, 71 (2006).

[45] T. Corbitt, Y. Chen, E. Innerhofer, H. Müller-Ebhardt, D. Ottaway, H. Rehbein, D. Sigg, S. Whitcomb, C. Wipf, and N. Mavalvala, Phys. Rev. Lett. 98, 150802 (2007).

[46] V. Braginsky, S. Strigin, and S. P. Vyatchanin, Phys. Lett. A 287, 331 (2001).

[47] G. J. Milburn, Quantum Semiclassic. Opt. 8, 269 (1996).

[48] A. C. Doherty, S. M. Tan, A. S. Parkins, and D. F. Walls, Phys. Rev. A 60, 2380 (1999).

[49] A. Hopkins, K. Jacobs, S. Habib, and K. Schwab, Phys. Rev. B 68, 235328 (2003).

[50] C. W. Gardiner and P. Zoller, Quantum Noise, 3rd ed. (Springer-Verlag, Berlin, 2004).

[51] A. Buonanno and Y. Chen, Phys. Rev. D 65, 042001 (2002).

[52] R. E. Kalman, J. Basic Eng. 82, 35 (1960).

[53] N. Wiener, Extrapolation, Interpolation, and Smoothing of Stationary Time Series (MIT Press, Cambridge, MA, 1949).

[54] H. Müller-Ebhardt, H. Rehbein, R. Schnabel, K. Danzmann, and Y. Chen, Phys. Rev. Lett. 100, 013601 (2008).

[55] H. Miao, S. Danilishin, H. Müller-Ebhardt, H. Rehbein, K. Somiya, and Y. Chen, e-print arXiv:0905.3729.

[56] A. Barchielli, Int. J. Theor. Phys. 32, 2221 (1992).

[57] L. Bouten, R. van Handel, and M. James, SIAM J. Control Optim. 46, 2199 (2007).

[58] C. M. Caves, Phys. Rev. D 23, 1693 (1981).

[59] A. Einstein, B. Podolsky, and N. Rosen, Phys. Rev. 47, 777 (1935).

[60] A. Furusawa, J. L. Soerensen, S. L. Braunstein, C. A. Fuchs, H. J. Kimble, and E. S. Polzik, Science 282, 706 (1998).

[61] W. P. Bowen, R. Schnabel, P. K. Lam, and T. C. Ralph, Phys. Rev. Lett. 90, 043601 (2003).

[62] G. Vidal and R. F. Werner, Phys. Rev. A 65, 032314 (2002).

[63] A. F. Pace, M. J. Collett, and D. F. Walls, Phys. Rev. A 47, 3173 (1993).

[64] A. Buonanno and Y. Chen, Phys. Rev. D 64, 042006 (2001).

[65] A. Buonanno and Y. Chen, Phys. Rev. D 67, 062002 (2003).

[66] http://www.ligo.mit.edu/bench/bench.html (Version 4.0)

[67] R. Adhikari (private communication).

[68] W. H. Zurek, S. Habib, and J. P. Paz, Phys. Rev. Lett. 70, 1187 (1993). 